\title{
Sensitive versus classical singular perturbation problem via Fourier transform
}

\author{
N. Meunier, E. Sanchez-Palencia ${ }^{\dagger}$
}

23rd February 2006

\begin{abstract}
We consider a class of singular perturbation elliptic boundary value problems depending on a parameter $\varepsilon$ which are classical for $\varepsilon>0$ but highly ill - posed for $\varepsilon=0$ as the boundary condition does not satisfy the Shapiro Lopatinskii condition. This kind of problems is motivated by certain situations in thin shell theory, but we only deal here with model problems and geometries allowing a Fourier transform treatment. We consider more general loadings and more singular perturbation terms than in previous works on the subject. The asymptotic process exhibits a complexification phenomenon: in some sense, the solution becomes more and more complicated as $\varepsilon$ decreases, and the limit does not exist in classical distribution theory (it only may be described in spaces of analytical functionals not enjoying localization properties). This phenomenon is associated with the emergence of the new characteristic parameter $|\log \varepsilon|$. Numerical experiments based on a formal asymptotics are presented, exhibiting features which are unusual in classical elliptic equations theory. We also give a Fourier transform treatment of classical singular perturbations in order to exhibit the drastic differences with the present situation.
\end{abstract}

\section{Introduction}

This paper is devoted to the theory and computation of a singular perturbation boundary value elliptic problem of the general variational form

$$
\left\{\begin{array}{l}
\text { Find } u^{\epsilon} \in V \text { such that, } \forall v \in V \\
a\left(u^{\epsilon}, v\right)+\epsilon^{2} b\left(u^{\epsilon}, v\right)=\langle f, v\rangle
\end{array}\right.
$$

where $a$ and $b$ are symmetric (or hermitian when complexes are involved) bilinear (or sesquilinear) forms, in the case when the limit problem (for $\varepsilon=0$ ) is an illposed elliptic problem in the following sense. It enjoys uniqueness properties (so

\footnotetext{
${ }^{*}$ Cérémade, Université Paris Dauphine. Place du Maréchal de Lattre de Tassigny 75775 Paris, France. (meunier@ceremade.dauphine.fr)

${ }^{\dagger}$ Laboratoire de Modélisation en Mécanique. Université Pierre et Marie Curie. 4, place Jussieu, 75252 Paris, France (sanchez@1mm.jussieu.fr)
} 
that $a(v, v)^{1 / 2}$ defines a norm on $V$ ) but the boundary conditions are such that they satisfy the Shapiro - Lopatinskii condition on a part $\Gamma_{0}$ of the boundary, but they do not satisfy it on the rest $\Gamma_{1}$ of the boundary. It follows that the limit problem is out of the general theory of elliptic boundary value problems. Let us recall that the Shapiro - Lopatinskii condition amounts to the impossibility of existence of local solutions (of the "frozen" problem at a point of the boundary) which are sinusoidal along the boundary and exponentially decaying in the normal coordinate towards the domain. It appears that the structure of the limit variational problem is drastically non classical. As a matter of fact, the energy space for the variational formulation of the limit problem is the completion of $V$ with the norm $a(v, v)^{1 / 2}$; this space, denoted by $V_{a}$ has a "very poor topology", it is very large and is not contained in the distribution space (see [12] and [5] in this connection). This remark is sufficient to foresee the unusual properties of the limit behavior. Indeed, the limit problem, considered as a variational one, only makes sense when the "loading" $f$ belongs to the dual $V_{a}^{\prime}$, which is obviously "very small", not containing the space $D$ of test functions of distributions. As a matter of fact, "almost any loading" $f$ is out of that space, so that only very special loadings give solutions with finite energy. This property motivates the term "sensitive" applied to that kind of problems (as singular perturbations). Moreover, the "very pathological" asymptotic behavior in "usual cases" when $f$ is not in $V_{a}^{\prime}$ remained open.

In recent times, it appeared that Fourier transform techniques in the direction tangent to the boundary bearing the "pathological boundary condition" allow in certain cases to understand why and how the solutions go out of the distribution space (see [18], [19] and [20]). In that papers, Fourier transform and computations "by hand" (or almost) with very special forms $a$ and $b$ give the asymptotic behavior using the fact that the Fourier transform of (general, not tempered) distributions are analytic functionals (not distributions) allowing to handle the limit problem and the asymptotic process for fairly general loadings $f$. The most striking property of that problem is the complexification phenomenon. As the limits are out of the distribution space, the sequences of solutions become more and more entangled as $\varepsilon$ decreases. But this process is very slow, in fact it is associated with the new parameter $|\log \varepsilon|$, which increases very slowly as $\varepsilon$ decreases; it describes the characteristic frequency of the main oscillations leading to the complexification. Then, a very simple heuristics gives a good description of $u^{\varepsilon}$ with small $\varepsilon$. The heuristics leads to a minimization problem in a space of dimension 1 with parameter $\xi$ ( $=$ the Fourier variable). Within the approximation of this heuristics, the solution enjoys interesting reciprocity properties concerning the point of application of the loading and the point of observation of the solution $u^{\varepsilon}$ (see Remark 13). Moreover, the complexification process is somewhat "non local": it is mainly localized in the vicinity of the boundary bearing the pathological boundary conditions, whatever the loading. This last point is very sticking, as it is in contradiction with classical regularity and wave front theory for elliptic equations in distribution theory. It is then apparent that such kind of phenomena send automatically out of distribution theory. 
This kind of singular perturbations appears in thin shell theory when the middle surface of the shell has everywhere both principal curvatures of the same sign and it is fixed or clamped by a part of its boundary and free by the rest. The boundary conditions on the free boundary do not satisfy the Shapiro - Lopatinskii condition (see for instance [15], Proposition VII 3.10, p. 238). In that case, $\varepsilon$ denotes the relative thickness of the shell, and the bilinear forms $a$ and $b$ are associated with the membrane and flection energies respectively. A complete understanding of these problems obviously involves general domains $\Omega$ and systems of equations with variable coefficients. Our aim is to generalize the previous results to more general solutions (in particular, "microlocal" versions of the Fourier transform are foreseen for general domains $\Omega$ ). But, for the time being, we only consider strip shaped domains and a very special elliptic equation with boundary conditions not satisfying the Shapiro - Lopatinskii condition.

Let us comment a little on Fourier transform of distributions. Usually [21], Fourier transforms are only defined for tempered distributions; this is very convenient, as the space $S^{\prime}$ of tempered distributions is transformed in itself by Fourier transform (i. e. the direct and inverse Fourier transforms define an isomorphism between $S_{x}^{\prime}$ and $S_{\xi}^{\prime}$ ). Nevertheless, it is possible to define (direct and inverse) Fourier transforms of $D^{\prime}$ [7] and [10]. The corresponding image is the space $Z^{\prime}$ and the Fourier transform (either direct or inverse, as they have analogous properties) define an isomorphism between $D^{\prime}$ and $Z^{\prime} . Z^{\prime}$ is the dual of the space of test functions $Z$, which is the Fourier image of the space of test functions $D$ of usual distributions. The space $Z$ is formed by the functions $\theta$ on $\mathbb{R}_{\xi}$ which are analytic (in fact they have analytic continuations) on the whole complex plane of the variable $\xi$ satisfying

$$
|\xi|^{q}|\theta(\xi)| \leq C_{q} e^{a_{q} \operatorname{Im}(\xi)}, \quad(q=0,1,2, \ldots)
$$

with $a$ and $C_{q}$ depending on the considered function $\theta$. This space is qualitatively very different of the space of test functions of distributions, $D$, which is formed by the function of class $C^{\infty}$ with compact support.

In other words, the (either direct or inverse) Fourier transforms of usual distributions are analytic functionals (i. e. functionals on a space of analytic functions) and more precisely, elements of $Z^{\prime}$. Obviously, this is mainly concerned with Fourier transforms of distributions which are not tempered; the Fourier transforms of tempered distributions are distributions (and tempered, in addition).

But there is a very serious drawback when passing from distributions to analytic functionals of $Z^{\prime}$. The success of distributions relies on the fact that they inherit very many properties of functions. In particular, distributions enjoy localization properties: the value of a distribution at a point does not make sense, but its action on any neighborhood of that point does (as the distribution may be tested on test functions with support in that neighborhood). Oppositely, in general, analytic functionals do not enjoy localization properties, as they only may act upon analytic functions (the support of which, by obvious analytic continuation properties is the whole $\mathbb{R}_{x}$ ). Theoretical considerations on this important property may be found in 
[13].

The motivation of this paper is two - fold. First, we generalize the methods and results of [20] (and of [18] and [19]) to more general loadings and equations. In particular, the perturbation is now "singular" in the classical sense that the order of differentiation of the limit problem is lower than that of the problems with $\varepsilon>0$. Moreover, concerning the formal asymptotics, it appears that it is very general, whatever the loading. This point is important, as the heuristics of the formal asymptotics is more or less evident for loadings applied on the boundary bearing the "pathological" boundary conditions (such as those considered in the above quoted literature), but it holds true in very general loadings, for which it is not evident. Similarly, the emergence of the new (moderately large) parameter $|\log \varepsilon|$ appears as fairly general. In any case, our aim is not to construct a general theory of that kind of singular perturbations, but rather to handle point examples helping us to understand the general structure of the asymptotic phenomenon in the very difficult framework of shells. In this context we obtained two asymptotic properties of the solution. The first one is the trend of the $a$ and $b$ energies to migrate towards the parts $\Gamma_{0}$ and $\Gamma_{1}$ of the boundary where the Shapiro - Lopatinskii condition is and is not satisfied, respectively (see Remark 4.2). The second one is a reciprocity property between the point of application of the loading and the point of observation of the solution (see Remark 4.3). The second motivation of this paper is to develop "classical" (i. e. non - sensitive) singular perturbations using the same kind of methods (Fourier transform) in order to allow easy comparisons with the sensitive case. Once more, we only consider some chosen examples, not trying to develop classical singular perturbation theory in the framework of Fourier transform. Up to our knowledge, the problems addressed in that part of the paper (Sections 5 and 6) are known. Our method (Fourier transform) seems to be new, and this gives a new insight allowing comparisons with the previous part. Accordingly, these two sections are written avoiding details which should often be redundant with the previous part. As a matter of fact, the convergence results (perhaps in other topologies) are well known when the loading is in the dual of the energy space of the limit problem, allowing a treatment by a priori estimates and variational theory (see [11] and [8]). In the case when the loading is not in $V_{a}^{\prime}$ it is known (see [17]) that local layers with unbounded energy appear in the vicinity of the singularities of the loading (i. e. the regions where the behavior of $f$ is not in $V_{a}^{\prime}$ ). That local layers were considered in [17] using a dilatation of the variable transversal to the layer. In the present approach, the Fourier transform gives directly information on the wavelengths which are relevant for small $\varepsilon$, describing directly the structure of the layer (see theorem 6.1 , which probably gives a new insight on this kind of layers).

Let us comment about a point that may be a little misleading for the reader. This paper contains mathematically rigorous parts as well as formal (heuristic) approximations in order to give understandable descriptions of the asymptotics and numerical computations of it. For instance, we often consider (hereafter $\delta$ denotes the Dirac mass) loadings $\delta\left(x_{1}\right) \delta\left(x_{2}-c\right)$ and $\delta\left(x_{1}\right) F\left(x_{2}\right)$ with $F \in L^{2}(0,1)$. The 
first one allows computations "by hand" which are exact and help us to understand the very structure of the asymptotics. The second one allows rigorous proofs of certain limit processes. In principle, there is no rigorous logical interaction between both patterns, even if the presentation mixes them a little. Another point is that, in order to avoid complexes in the Fourier transforms (and then simplify the computations and figures), we only deal with real even functions, which have (direct and inverse) real and even Fourier transforms. In particular, the loadings are mainly $\delta\left(x_{1}\right)$-like but obviously, solutions for other loadings may immediately written by convolution. Accordingly, real expressions are sometimes written instead of complex expressions. This allows us to interpret certain variational formulations in terms of minimization of real functionals.

The paper is organized as follows. Sections 2 contains a study of the limit problem (with "pathological" boundary conditions) via Fourier transform. It only differs from sect 2 of [20] in the applied loading. Section 3 contains the singular perturbation problem and the convergence theorems of the solutions and of their Fourier transforms. For obvious reasons the topology of these convergences is "very poor" and an approximate description of the solution with small $\varepsilon$ is necessary to have a good insight of the asymptotic phenomenon. This is done in Section 4. The "classical case" when the boundary conditions satisfy the Shapiro - Lopatinskii condition are addressed in Sections 5 and 6, the former contains the limit problem and the latter, the singular perturbation.

Notations are standard. We denote

$$
\partial_{k}=\frac{\partial}{\partial x_{k}}, \quad k=1,2 .
$$

It should be noted that if $u$ is a function that only depends on $x_{2}, \partial_{2}$ will also denote the derivative $u^{\prime}\left(x_{2}\right)$.

As several (equivalent) definitions of the Fourier transform are found in current literature, we should specify that we use

$$
\hat{f}(\xi)=\int_{-\infty}^{+\infty} f(x) e^{-i \xi x} d x \quad \text { and } \quad f(x)=\frac{1}{2 \pi} \int_{-\infty}^{+\infty} \hat{f}(\xi) e^{i \xi x} d \xi
$$

As a consequence, the Fourier transform of the Dirac mass $\delta(x)$ is $1_{\xi}$ (i. e., the function of $\xi$ equal to 1 ), whereas the inverse Fourier transform of $\delta(\xi)$ is $(2 \pi)^{-1} 1_{x}$. For the sake of simplicity and without essential restriction, we shall mainly deal with real and even functions, which have real and even Fourier transforms. 


\section{An elliptic problem with "unadapted" boundary condi- tions}

Let $\Omega=\mathbb{R} \times(0,1)$ be the infinite strip in the $\mathbb{R}^{2}$ plane of the variable $x=\left(x_{1}, x_{2}\right)$ and let $a$ be the bilinear form given by:

$$
a(u, v)=\int_{\Omega} \triangle u \triangle v \mathrm{~d} x .
$$

We consider the following variational problem

$$
\left\{\begin{array}{l}
\text { Find } u \in V_{a} \text { such that, } \forall v \in V_{a} \\
a(u, v)=\langle f, v\rangle
\end{array}\right.
$$

where the space $V_{a}$ is the "energy space" with the essential boundary conditions

$$
v\left(x_{1}, 0\right)=\partial_{2} v\left(x_{1}, 0\right)=0,
$$

which is defined as the completion with the norm $\|v\|_{a}=a(v, v)$ of the set of $H^{2}(\Omega)$ functions satisfying (2.3), while $f$ is an element of the dual, denoted by $V_{a}^{\prime}$.

This problem exhibits several special features which we give as remarks.

Remark 1 . We note that the energy space $V_{a}$ is not a classical space. In fact, $\|v\|_{a}$ is a norm on $H^{2}(\Omega)$ (or any other space of sufficiently regular functions) with the essential boundary conditions (2.3). Indeed, when it vanishes, we have $\Delta v=0$ with (2.3). This amounts to the Cauchy problem for the laplacian, which classically enjoys uniqueness (from the Holmgren local uniqueness theorem together with analytic continuation, see for instance [2]). Then, $V_{a}$ is well defined in a somewhat abstract way. But obviously the Cauchy elliptic problems are ill - posed (so that "very large" $v$ may correspond to "very small" $\Delta v$, see for instance [2] or [6]). In fact, $V_{a}$ is a "very large space" not contained in the distribution space $D^{\prime}(\Omega)$ (see [12] and [5]). This point will not be explicitly addressed here, but it will be (more or less) apparent from the forthcoming developments.

Remark 2. The bilinear form a given by (2.1) is not classical, as it involves the block $\Delta v$ in $L^{2}$, not each second order derivative. Compare with section 5, (5.1); both expressions are not equivalent when the essential boundary conditions (2.3) are not prescribed all along the boundary of the domain.

Remark 3. Since $a$ is a symmetric form, it follows that when the loading $f$ is in the dual space $V_{a}^{\prime}$, problem (2.2) is equivalent to the minimization of the functional:

$$
\int_{\Omega}(\triangle v)^{2} \mathrm{~d} x-2 \int_{\Omega} f v \mathrm{~d} x
$$

over $V_{a}$. 
Moreover, after a formal integration by parts, we easily deduce that the classical formulation of problem (2.2) is :

$$
\left\{\begin{array}{l}
\triangle^{2} u=f \text { on } \Omega \\
u\left(x_{1}, 0\right)=\partial_{2} u\left(x_{1}, 0\right)=0, \forall x_{1} \in \mathbb{R} \\
\triangle u\left(x_{1}, 1\right)=\partial_{2} \triangle u\left(x_{1}, 1\right)=0, \forall x_{1} \in \mathbb{R} .
\end{array}\right.
$$

Remark 4. We note that under this "classical" ( = non variational) form, the problem makes sense for more general loadings $f$, not necessarily contained in $V_{a}^{\prime}$. We shall take it of the form

$$
f\left(x_{1}, x_{2}\right)=\delta\left(x_{1}\right) F\left(x_{2}\right)
$$

which allows, by convolution in $x_{1}$, very general loadings.

Remark 5. The boundary value problem (2.5) is not classical. It involves the new natural boundary conditions on $x_{2}=1$, but they do not satisfy the Shapiro - Lopatinskii condition (see for instance [1] or [3]). Indeed, considering the upper half plane $\left(x_{1}, x_{2}\right), x_{2} \geq 0$, and the equation

$$
\triangle^{2} u\left(x_{1}, x_{2}\right)=\left(\partial_{1}^{4}+2 \partial_{1}^{2} \partial_{2}^{2}+\partial_{2}^{4}\right) u\left(x_{1}, x_{2}\right)=0,
$$

with the boundary conditions

$$
\triangle u\left(x_{1}, 0\right)=\partial_{2} \triangle u\left(x_{1}, 0\right)=0,
$$

and, taking the Fourier transform in the $x_{1}$ direction, we get:

$$
\left(\xi^{4}-2 \xi^{2} \partial_{2}^{2}+\partial_{2}^{4}\right) \hat{u}\left(\xi, x_{2}\right)=0,
$$

with the boundary conditions

$$
\left(\xi^{2}-\partial_{2}^{2}\right) \hat{u}(\xi, 1)=\partial_{2}\left(\xi^{2}-\partial_{2}^{2}\right) \hat{u}(\xi, 1)=0 .
$$

It then appears that this problem has non zero-solutions, such as $\hat{u}=A e^{-|\xi| x_{2}}$. This means that the Shapiro - Lopatinskii condition is not satisfied. Therefore, it is not a classical problem and it has no solution in general.

In order to avoid problems explained in Remark 5, we will first consider the $x_{1}$ Fourier transform of the previous boundary value problem (2.5) with a load given by (2.6). The new problem is an ODE, which depends on a parameter $\xi$ and which has solutions for any value of the parameter. Next, taking the inverse Fourier transform, we shall obtain solutions of (2.5) in the space of analytical functionals $Z^{\prime}$. In that case, using the Fourier transform of (2.5) with respect to $x_{1}$ and denoting the Fourier transform of $u\left(x_{1}, x_{2}\right)$ by $\hat{u}\left(\xi, x_{2}\right)$, we obtain the following boundary value problem for $x_{2} \in(0,1)$, which depends on the parameter $\xi \in \mathbb{R}$

$$
\left\{\begin{array}{l}
\left(\partial_{2}^{2}-\xi^{2}\right)\left(\partial_{2}^{2}-\xi^{2}\right) \hat{u}\left(\xi, x_{2}\right)=F\left(x_{2}\right), \forall x_{2} \in(0,1) \\
\hat{u}(\xi, 0)=\partial_{2} \hat{u}(\xi, 0)=0 \\
\left(\partial_{2}^{2}-\xi^{2}\right) \hat{u}(\xi, 1)=\partial_{2}\left(\partial_{2}^{2}-\xi^{2}\right) \hat{u}(\xi, 1)=0
\end{array}\right.
$$


In order to get solutions as explicit as possible, we shall begin with $F\left(x_{2}\right)=$ $\delta\left(x_{2}-c\right), 0<c<1$, where $\delta$ denotes the Dirac mass, this gives

$$
f=\delta\left(x_{1}\right) \delta\left(x_{2}-c\right) .
$$

We note that it belongs to $H^{-2}(0,1)$.

In that case, the boundary value problem (2.7) becomes

$$
\left\{\begin{array}{l}
\left(\partial_{2}^{2}-\xi^{2}\right)\left(\partial_{2}^{2}-\xi^{2}\right) \hat{u}\left(\xi, x_{2}\right)=\delta\left(x_{2}-c\right), \forall x_{2} \in(0,1) \\
\hat{u}(\xi, 0)=\partial_{2} \hat{u}(\xi, 0)=0 \\
\left(\partial_{2}^{2}-\xi^{2}\right) \hat{u}(\xi, 1)=\partial_{2}\left(\partial_{2}^{2}-\xi^{2}\right) \hat{u}(\xi, 1)=0
\end{array}\right.
$$

Recalling first that solutions of

$$
\left(\partial_{2}^{2}-\xi^{2}\right)\left(\partial_{2}^{2}-\xi^{2}\right) \hat{u}\left(\xi, x_{2}\right)=0,
$$

are linear combinations of

$$
e^{|\xi| x_{2}}, e^{-|\xi| x_{2}}, x_{2} e^{|\xi| x_{2}}, x_{2} e^{-|\xi| x_{2}}
$$

we deduce that solutions of (2.9) are functions $\hat{u}\left(\xi, x_{2}\right)$ of the form

$$
\hat{u}\left(\xi, x_{2}\right)=\left\{\begin{array}{lll}
\hat{u}^{-}\left(\xi, x_{2}\right) & \text { if } & x_{2} \in(0, c), \\
\hat{u}^{+}\left(\xi, x_{2}\right) & \text { if } & x_{2} \in(c, 1),
\end{array}\right.
$$

where $\hat{u}^{-}(\xi,),. \hat{u}^{+}(\xi,$.$) are defined on \mathbb{R}$ (i. e., we shall also consider their analytic continuation out of their definition domain) such that

$$
\left\{\begin{array}{l}
\hat{u}^{-}\left(\xi, x_{2}\right)=\left(C_{1}^{-}+C_{3}^{-} x_{2}\right) e^{|\xi| x_{2}}+\left(C_{2}^{-}+C_{4}^{-} x_{2}\right) e^{-|\xi| x_{2}} \\
\hat{u}^{+}\left(\xi, x_{2}\right)=\left(C_{1}^{+}+C_{3}^{+} x_{2}\right) e^{|\xi| x_{2}}+\left(C_{2}^{+}+C_{4}^{+} x_{2}\right) e^{-|\xi| x_{2}}
\end{array}\right.
$$

where $C_{k}^{-}, C_{k}^{+} \in \mathbb{C}$, for $k \in\{1,2,3,4\}$. The distribution $\delta\left(x_{2}-c\right)$ at the right hand side of (2.9) may be described in terms of the jumps and we see that $\hat{u}$ is a solution of

$$
\left(\partial_{2}^{2}-\xi^{2}\right)\left(\partial_{2}^{2}-\xi^{2}\right) \hat{u}\left(\xi, x_{2}\right)=\delta\left(x_{2}-c\right)
$$

when $\hat{u}^{-}$and $\hat{u}^{+}$satisfy

$$
\left\{\begin{aligned}
\hat{u}^{-}(\xi, c) & =\hat{u}^{+}(\xi, c) \\
\left(\hat{u}^{-}\right)^{\prime}(\xi, c) & =\left(\hat{u}^{+}\right)^{\prime}(\xi, c) \\
\left(\hat{u}^{-}\right)^{\prime \prime}(\xi, c) & =\left(\hat{u}^{+}\right)^{\prime \prime}(\xi, c) \\
\left(\hat{u}^{-}\right)^{\prime \prime \prime}(\xi, c)+1 & =\left(\hat{u}^{+}\right)^{\prime \prime \prime}(\xi, c)
\end{aligned}\right.
$$

where $\left(\hat{u}^{-}\right)^{\prime},\left(\hat{u}^{-}\right)^{\prime \prime},\left(\hat{u}^{-}\right)^{\prime \prime \prime}$ denote $\partial_{2}\left(\hat{u}^{-}\right), \partial_{2}^{2}\left(\hat{u}^{-}\right), \partial_{2}^{3}\left(\hat{u}^{-}\right)$respectively. A direct computation on the derivatives of $\hat{u}^{-}$and $\hat{u}^{+}$shows that

$$
\left\{\begin{array}{l}
C_{4}^{+}=C_{4}^{-}+\frac{e^{|\xi| c}}{4 \xi^{2}} \\
C_{3}^{+}=C_{3}^{-}+\frac{e^{-|\xi| c}}{4 \xi^{2}} \\
C_{2}^{+}=C_{2}^{-}+\frac{(1-|\xi| c) e^{|\xi| c}}{4|\xi|^{3}} \\
C_{1}^{+}=C_{1}^{-}-\frac{\left(1+|\xi| c e^{-|\xi| c}\right.}{4 \mid \xi^{3}}
\end{array}\right.
$$


Referring next to the boundary conditions of (2.9), it yields

$$
\left\{\begin{array}{l}
C_{4}^{-}=-\frac{e^{|\xi| c}}{4 \xi^{2}} \\
C_{3}^{-}=-\frac{e^{-|\xi| c}}{4 \xi^{2}} \\
C_{2}^{-}=-\frac{1}{4|\xi|^{3}} \cosh (|\xi| c), \\
C_{1}^{-}=\frac{1}{4|\xi|^{3}} \cosh (|\xi| c) .
\end{array}\right.
$$

Therefore, we obtain

$$
\hat{u}^{-}\left(\xi, x_{2}\right)=\frac{1}{2|\xi|^{3}} \cosh (|\xi| c) \sinh \left(|\xi| x_{2}\right)-\frac{x_{2}}{2 \xi^{2}} \cosh \left(|\xi|\left(x_{2}-c\right)\right)
$$

and

$$
\begin{aligned}
\hat{u}^{+}\left(\xi, x_{2}\right)= & \hat{u}^{-}\left(\xi, x_{2}\right)+\frac{\left(x_{2}-c\right)}{2 \xi^{2}} \cosh \left(|\xi|\left(x_{2}-c\right)\right) \\
& -\frac{1}{2|\xi|^{3}} \sinh \left(|\xi|\left(x_{2}-c\right)\right),
\end{aligned}
$$

so that the solution $\hat{u}\left(\xi, x_{2}\right)$ is given by (2.11) with (2.17) and (2.18).

Remark 6. It should be noted that this solution is analytical of $\xi \in \mathbb{R}$; the denominators involving powers of $|\xi|$ are misleading. In fact they disappear when developing the expressions. Moreover, $\hat{u}(\xi, \bullet)$ can be considered as an analytic function of $\xi \in \mathbb{R}$ with values in $H^{2}(0,1)$. This follows easily from the analyticity with respect to $\xi$ of the bilinear form associated with the boundary value problem (2.9), (see [9] and more precisely [14] sect. V.4 if necessary).

We note that for all $\left.x_{2} \in\right] 0,1\left[\right.$, with $x_{2} \neq c$, and for $|\xi| \rightarrow+\infty$, we have

$$
\hat{u}\left(\xi, x_{2}\right) \approx \frac{1}{8|\xi|^{3}} e^{\left(c+x_{2}\right)|\xi|} .
$$

Since the behavior of $\hat{u}$ is at infinity exponential, it means that $\hat{u}$ is a non tempered distribution. The solution $u\left(x_{1}, x_{2}\right)$ of the original problem in $\left(x_{1}, x_{2}\right)$ may be defined as the inverse Fourier transform of $\hat{u}\left(\xi, x_{2}\right)$.This Fourier transform $\hat{u}\left(\xi, x_{2}\right)$ is in $D^{\prime}$ but not in $S^{\prime}$ (see the Introduction). Therefore, problem (2.5) has a unique solution defined by its Fourier transform $\hat{u}$. Moreover, $u$ itself is not a distribution, but a function of $x_{2}$ with values in the space of analytic functionals $Z^{\prime}\left(\mathbb{R}_{x_{1}}\right)$. It should also be mentioned, for ulterior utilization, the obvious fact that $\hat{u}$ is $O(1)$ on finite intervals of $\xi$.

As we shall see in the second part of this article (sections 5 and 6 ), this situation is very different from the one when the Shapiro - Lopatinskii condition is satisfied. Compare for instance (2.19) with (5.22) - (5.24). Moreover, we also see in (2.19) that the coefficient of the exponential function increases with $x_{2}$. It means that the corresponding singular behavior is more important for $x_{2}>c$ than for $x_{2}=c$ (i. e., the singular behavior is maximal at the boundary $\Gamma_{1}$, not at the point where the 
loading is applied. This behavior, which does not agree with classical theory of elliptic partial differential equations is exhibited in Fig. 4 hereafter.

In the sequel, we will consider $\hat{u}\left(\xi, x_{2}\right)$ as a function of $\xi$ and $x_{2}$ or as a function $\hat{u}(\xi)$, that depends on a parameter $\xi$, and which is a function of $x_{2}$.

Remark 7. There is another streaking feature of the asymptotic structure of $\hat{u}(\xi)$ with large $|\xi|$. By inspection of (2.17) and (2.18) it is apparent that, up to exponentially negligible terms (for $|\xi| \rightarrow \infty$ ) $\hat{u}$ is merely $\hat{u}^{-}$, where we even may only consider the first term in (2.17). In other words, within this approximation, the folding at $x_{2}=c$ disappears and the function is also analytic in $x_{2}$.

Remark 8. In the case when the loading is given by:

$$
f\left(x_{1}, x_{2}\right)=\delta\left(x_{1}\right) F\left(x_{2}\right)
$$

with $F \in L^{2}(0,1)$, the Fourier transform $\hat{u}\left(\xi, x_{2}\right)$ may be constructed as above. Concerning its behavior as $|\xi| \rightarrow \infty$, a simple superposition argument shows that for $\left.x_{2} \in\right] 0,1[$, and for $|\xi| \rightarrow+\infty$,

$$
K(c) e^{\left(c+x_{2}\right)|\xi|} \leq\left|\hat{u}\left(\xi, x_{2}\right)\right| \leq K_{2} e^{\left(c_{2}+x_{2}\right)|\xi|},
$$

where $c$ denotes any value in $\operatorname{support}(F)$ and $c_{2}$ is the upper extremity of that support. Consequently, the behavior of $\hat{u}\left(\bullet, x_{2}\right)$ at infinity is exponential and $\hat{u}\left(\bullet, x_{2}\right)$ is a non tempered distribution. Furthermore, using again a convolution argument, the conclusion is also valid for general data (provided their Fourier transform do not vanish exponentially as $|\xi| \rightarrow+\infty$ ).

\section{Singular perturbation with "unadapted boundary con- ditions" in the limit problem}

Let us now consider the variational problem depending on the parameter $\varepsilon>0$ given by

$$
\left\{\begin{array}{l}
\text { Find } u^{\epsilon} \in V \text { such that, } \forall v \in V \\
a\left(u^{\epsilon}, v\right)+\epsilon^{2} b\left(u^{\epsilon}, v\right)=\langle f, v\rangle
\end{array}\right.
$$

where $a$ is still given by (2.1) and $b$ is such that

$$
b(u, v)=\int_{\Omega}\left(\partial_{1}^{p} u \partial_{1}^{p} v+\partial_{2}^{p} u \partial_{2}^{p} v\right) \mathrm{d} x,
$$

for some integer $p>2$. In the sequel, we will take $p=3$ without loss of generalty.

In this case, the energy space $V$ is the completion of $H^{3}(\Omega)$ functions $v$ satisfying the essential boundary conditions

$$
v\left(x_{1}, 0\right)=\partial_{2} v\left(x_{1}, 0\right)=\partial_{2}^{2} v\left(x_{1}, 0\right)=0,
$$

with the norm $\|v\|_{V}^{2}=a(v, v)+b(v, v)$. 
Let $H_{\Gamma_{0}}^{3}(0,1)$ and $H_{\Gamma_{0}}^{2}(0,1)$ be defined as follows

$$
\begin{aligned}
& H_{\Gamma_{0}}^{3}(0,1)=\left\{v \in H^{3}(0,1), \text { s.t. } v(0)=\partial_{2} v(0)=\partial_{2}^{2} v(0)=0\right\} \\
& H_{\Gamma_{0}}^{2}(0,1)=\left\{v \in H^{2}(0,1), \text { s.t. } v(0)=\partial_{2} v(0)=0\right\}
\end{aligned}
$$

Remark 9. Due to Poincaré inequality (used three times), the identity between spaces,

$$
V=L^{2}\left(\mathbb{R}_{x_{1}} ; H_{\Gamma_{0}}^{3}(0,1)_{x_{2}}\right) \cap H^{3}\left(\mathbb{R}_{x_{1}} ; L^{2}(0,1)_{x_{2}}\right),
$$

is easily proved.

Moreover, using again Poincaré's inequality, we have

$$
C\|v\|_{V}^{2} \geq a(v, v)+\epsilon^{2} b(v, v) \geq c \epsilon^{2}\|v\|_{V}^{2}, \text { for all } v \in V,
$$

so that the constant of coerciveness tends to zero as $\epsilon$ goes to zero.

We shall develop the rest of this section in the case of the loading $f\left(x_{1}, x_{2}\right)=$ $\delta\left(x_{1}\right) F\left(x_{2}\right)$ with $F \in L^{2}(0,1)$. Obviously, this $f$ is in $V^{\prime}$ (the dual of $V$ ) so that the existence and uniqueness of the solution is ensured for $\varepsilon>0$. For $\varepsilon=0$ the problem was considered in the previous section (see Remark 8).

From the previous variational formulation (3.1), the classical formulation is deduced by an integration by parts:

$$
\triangle^{2} u^{\epsilon}-\epsilon^{2}\left(\partial_{1}^{6}+\partial_{2}^{6}\right) u^{\epsilon}=f \text { on } \Omega
$$

with the (essential) boundary conditions on $x_{2}=0$ :

$$
u^{\epsilon}\left(x_{1}, 0\right)=\partial_{2} u^{\epsilon}\left(x_{1}, 0\right)=\partial_{2}^{2} u^{\epsilon}\left(x_{1}, 0\right)=0, \forall x_{1} \in \mathbb{R}
$$

and the (natural) boundary conditions on $x_{2}=1$ :

$\epsilon^{2} \partial_{2}^{3} u^{\epsilon}\left(x_{1}, 1\right)=\left(\triangle-\epsilon^{2} \partial_{2}^{4}\right) u^{\epsilon}\left(x_{1}, 1\right)=-\partial_{2}\left(\triangle-\epsilon^{2} \partial_{2}^{4}\right) u^{\epsilon}\left(x_{1}, 1\right)=0, \forall x_{1} \in \mathbb{R}$.

Remark 10. The previous perturbation is singular in the classical sense since the new added term contains derivatives of order 6 , which is larger than 4 for the unperturbed problem. Moreover, the perturbed problem is elliptic. Indeed, the principal order is $-\epsilon^{2}\left(\partial_{1}^{6}+\partial_{2}^{6}\right)$, so that, the principal symbol is $\epsilon^{2}\left(\xi_{1}^{6}+\xi_{2}^{6}\right)$, which only vanishes for $\xi_{\alpha}=0$. The boundary conditions on $x_{1}=0$ are the Dirichlet ones, which obviously satisfy the Shapiro -Lopatinskii condition. Furthermore, the perturbed problem satisfies the Shapiro - Lopatiskii condition in $x_{2}=1$. To see this, let us consider again the principal symbol and let $\xi_{1} \in \mathbb{R}$, $\xi_{2} \in \mathbb{C}$ be such that $\xi_{1}^{6}+\xi_{2}^{6}=0$. The corresponding local solution takes the form $u=e^{\xi_{1} x_{1}}\left(\sum_{i=1}^{3} A_{i} e^{\alpha_{i} x_{2}}\right)$, where $\alpha_{i}^{6}=-\xi_{1}^{6}$ and the imaginary part of $\alpha_{i}$ is negative. Then, prescribing the natural boundary conditions (which correspond to the principal term of (3.9)) $\epsilon^{2} \partial_{2}^{3} u\left(x_{1}, 1\right)=-\epsilon^{2} \partial_{2}^{4} u\left(x_{1}, 1\right)=\epsilon^{2} \partial_{2}^{5} u\left(x_{1}, 1\right)=0$, $\forall x_{1} \in \mathbb{R}$, hence $u=0$. 
Let us now consider the $x_{1}$ Fourier transform of the previous problem. First, we introduce some notations. For $u, v$ in $H^{2}(0,1)$, let $\hat{a}$ be defined by:

$$
\hat{a}(u, v)=\left\langle\left(\partial_{2}^{2}-\xi^{2}\right) u,\left(\partial_{2}^{2}-\xi^{2}\right) v\right\rangle
$$

and similarly, for $u, v$ in $H^{3}(0,1)$, let $\hat{b}$ be the form defined by

$$
\hat{b}(u, v)=|\xi|^{6}\langle u, v\rangle+\left\langle\partial_{2}^{3} u, \partial_{2}^{3} v\right\rangle
$$

where $\langle$,$\rangle denotes the usual scalar product in L^{2}(0,1)$.

Remark 11. It should be noted that $\hat{a}$ and $\hat{b}$ depend on $\xi$, but for simplicity, we will not mention it in the notation when no confusion arises.

We shall consider here a given force of the form $f\left(x_{1}, x_{2}\right)=\delta\left(x_{1}\right) F\left(x_{2}\right)$ with $F \in L^{2}(0,1)$. We shall first give the $x_{1}$ Fourier transform of the classical problem (3.7), (3.8) and (3.9) equivalent to the $x_{1}$ Fourier transform of the variational problem (3.1).

Taking the $x_{1}$ Fourier transform of (3.7), (3.8) and (3.9), we obtain the (dependent on the parameter $\xi$ ) ordinary differential equation for $x_{2} \in(0,1)$ :

$$
\left(\left(\partial_{2}^{2}-\xi^{2}\right)\left(\partial_{2}^{2}-\xi^{2}\right)-\epsilon^{2}\left(-\xi^{6}+\partial_{2}^{6}\right)\right) \hat{u}^{\epsilon}\left(\xi, x_{2}\right)=F\left(x_{2}\right), \forall x_{2} \in(0,1)
$$

with the boundary conditions

$$
\begin{aligned}
\hat{u}^{\epsilon}(\xi, 0) & =\partial_{2} \hat{u}^{\epsilon}(\xi, 0)=\partial_{2}^{2} \hat{u}^{\epsilon}(\xi, 0)=0 \\
\epsilon^{2} \partial_{2}^{3} \hat{u}^{\epsilon}(\xi, 1) & =\left(\left(\partial_{2}^{2}-\xi^{2}\right)-\epsilon^{2} \partial_{2}^{4}\right) \hat{u}^{\epsilon}(\xi, 1) \\
& =-\partial_{2}\left(\left(\partial_{2}^{2}-\xi^{2}\right)-\epsilon^{2} \partial_{2}^{4}\right) \hat{u}^{\epsilon}(\xi, 1)=0 .
\end{aligned}
$$

As we shall see, this is a classical Lax - Milgram boundary value problem; its variational formulation, which follows from standard integration by parts is:

$$
\left\{\begin{array}{l}
\text { Find } \hat{u}^{\epsilon}(\xi) \in H_{\Gamma_{0}}^{3}(0,1) \text { such that, } \forall v \in H_{\Gamma_{0}}^{3}(0,1) \\
\hat{a}\left(\hat{u}^{\epsilon}(\xi), v\right)+\epsilon^{2} \hat{b}\left(\hat{u}^{\epsilon}(\xi), v\right)=\langle F, v\rangle .
\end{array}\right.
$$

The rest of this section is organized as follows. First, for all fixed and finite value of $\xi$, we prove the existence and uniqueness of the solution of problem (3.15). Next, we shall prove the convergence of the sequence $\left(\hat{u}^{\epsilon}(\xi)\right)_{\epsilon}$ of solutions of (3.15) to $\hat{u}(\xi)$ solution of (2.7), as $\epsilon$ goes to zero, for fixed value of $\xi$. Then, we shall prove the same convergence in terms of distributions on $\mathbb{R}_{\xi}$, and we shall deduce the convergence of the $u^{\varepsilon}$ to the solution $u$ of the limit problem for $\varepsilon=0$ of the previous section in terms of analytical functionals on $\mathbb{R}_{x}$.

Lemma 3.1. There exists $C>0$ such that for all $u$ in $H_{\Gamma_{0}}^{2}(0,1)$, we have

$$
\left\|\left(\partial_{2}^{2}-\xi^{2}\right) u\right\|_{L^{2}}^{2} \geq C\|u\|_{H^{2}}^{2},
$$

where $C$ can be chosen independently of $\xi$ on bounded intervals of $\mathbb{R}_{\xi}$. 
Lemma 3.2. For $\epsilon>0$ there exists $C>0$ such that for all $u$ in $H_{\Gamma_{0}}^{3}(0,1)$, we have

$$
\hat{a}(u, u)+\epsilon^{2} \hat{b}(u, u) \geq C \epsilon^{2}\|u\|_{H^{3}}^{2},
$$

where $C$ can be chosen independently of $\xi$ on bounded intervals of $\mathbb{R}_{\xi}$.

It follows from the two previous lemmas that (3.15) with $\varepsilon=0$ and $\varepsilon>0$ are classical variational problems in $H_{\Gamma_{0}}^{2}(0,1)$ and $H_{\Gamma_{0}}^{3}(0,1)$ respectively (note that the right hand sides define continuous functionals on both spaces), so that the solutions $\hat{u}(\xi)$ and $\hat{u}^{\varepsilon}(\xi)$ are well defined and unique by the Lax - Milgram theorem.

Proof of Lemma 3.1. Let $\xi$ be fixed, and let us consider $\left\|\left(\partial_{2}^{2}-\xi^{2}\right) u\right\|_{L^{2}}^{2}$ for $u$ in $H_{\Gamma_{0}}^{2}(0,1)$. In order to establish the coerciveness character of the previous expression, using the variation of parameter method, we solve for some given $\varphi \in$ $L^{2}(0,1)$ the following equation:

$$
\left(\partial_{2}^{2}-\xi^{2}\right) w=\varphi, \text { with } w(0)=\partial_{2} w(0)=0 .
$$

We observe that $w$ is such that $w\left(x_{2}\right)=A\left(x_{2}\right) e^{|\xi| x_{2}}+B\left(x_{2}\right) e^{-|\xi| x_{2}}$, where the functions $A$ and $B$ can be chosen such that

$$
\left\{\begin{array}{l}
A^{\prime}\left(x_{2}\right) e^{|\xi| x_{2}}+B^{\prime}\left(x_{2}\right) e^{-|\xi| x_{2}}=0 \\
|\xi|\left(A^{\prime}\left(x_{2}\right) e^{|\xi| x_{2}}-B^{\prime}\left(x_{2}\right) e^{-|\xi| x_{2}}\right)=\varphi\left(x_{2}\right)
\end{array}\right.
$$

hence,

$$
A^{\prime}\left(x_{2}\right)=\frac{\varphi\left(x_{2}\right)}{2|\xi|} \text { and } B^{\prime}\left(x_{2}\right)=-\frac{\varphi\left(x_{2}\right)}{2|\xi|} .
$$

Consequently, referring to the boundary conditions, $w(0)=\partial_{2} w(0)=0$, this gives

$$
A\left(x_{2}\right)=\int_{0}^{x_{2}} \frac{\varphi(y)}{2|\xi|} \mathrm{d} y \text { and } B\left(x_{2}\right)=-\int_{0}^{x_{2}} \frac{\varphi(y)}{2|\xi|} \mathrm{d} y
$$

so that the solution $w\left(x_{2}\right)$ writes

$$
w\left(x_{2}\right)=\left[x_{2} \int_{0}^{x_{2}} \varphi(y) d y\right] \frac{\sinh \left(|\xi| x_{2}\right)}{|\xi| x_{2}} .
$$

This expression is analytic for real $|\xi| x_{2}$ with values in $H_{\Gamma_{0}}^{2}$, and the conclusion follows.

The proof of Lemma 3.2 is immediate using merely the last term in (3.11) and Poincaré's inequality three times.

The convergence of the Fourier transforms with fixed $\xi$ is stated as follows: 
Lemma 3.3. Let $\xi$ be fixed and $\hat{u}^{\epsilon}(\xi), \hat{u}(\xi)$ be the solutions of (3.15) and (2.7) respectively with the loading (2.20), then we have:

$$
\hat{u}^{\epsilon}(\xi) \rightarrow \hat{u}(\xi) \text { strongly in } H_{\Gamma_{0}}^{2}(0,1) \text {, as } \epsilon \text { goes to zero. }
$$

Proof of Lemma 3.3. Let $\xi$ be fixed. We first prove the weak convergence.

Let us take $\hat{u}^{\epsilon}(\xi)$ as a test function in (3.15), we obtain

$$
\left\|\left(\partial_{2}^{2}-\xi^{2}\right) \hat{u}^{\epsilon}(\xi)\right\|_{L^{2}}^{2}+\epsilon^{2}\left(\xi^{6}\left\|\hat{u}^{\epsilon}(\xi)\right\|_{L^{2}}^{2}+\left\|\partial_{2}^{3} \hat{u}^{\epsilon}(\xi)\right\|_{L^{2}}^{2}\right)=\left\langle F, \hat{u}^{\epsilon}(\xi)\right\rangle,
$$

therefore,

$$
\left\|\left(\partial_{2}^{2}-\xi^{2}\right) \hat{u}^{\epsilon}(\xi)\right\|_{L^{2}}^{2} \leq\left\langle F, \hat{u}^{\epsilon}(\xi)\right\rangle
$$

next, using Lemma 3.1 and majorizing the right hand side term with Cauchy's inequality, we get

$$
\left\|\hat{u}^{\epsilon}(\xi)\right\|_{H^{2}} \leq C\|F\|_{L^{2}} .
$$

Consequently, the sequence $\left(\hat{u}^{\epsilon}(\xi)\right)_{\epsilon}$ remains bounded in $H_{\Gamma_{0}}^{2}(0,1)$ as $\epsilon$ goes to zero, and up to a subsequence that we do not relabel, this gives the existence of $\hat{u}^{*}(\xi)$ in $H_{\Gamma_{2}}^{2}(0,1)$ such that

$$
\hat{u}^{\epsilon}(\xi) \rightarrow \hat{u}^{*}(\xi) \text { weakly in } H_{\Gamma_{0}}^{2}(0,1) .
$$

Let us now prove that $\hat{u}^{*}(\xi)$ is the solution of the following variational problem (which is nothing but the variational formulation of the limit problem (2.7)):

$$
\left\{\begin{array}{l}
\text { Find } \hat{u}(\xi) \in H_{\Gamma_{0}}^{2}(0,1) \text { such that, } \forall v \in H_{\Gamma_{0}}^{2}(0,1) \\
\left\langle\left(\partial_{2}^{2}-\xi^{2}\right) \hat{u}^{\epsilon}(\xi),\left(\partial_{2}^{2}-\xi^{2}\right) v\right\rangle=\langle F, v\rangle .
\end{array}\right.
$$

Using the uniqueness of the solution of problem (3.27), this will give the convergence of the whole sequence.

Recalling (3.25), we see that

$$
\left\|\hat{u}^{\epsilon}(\xi)\right\|_{L^{2}} \leq C
$$

hence, referring to (3.23), we obtain

$$
\left\|\partial_{2}^{3} \hat{u}^{\epsilon}(\xi)\right\|_{L^{2}} \leq \frac{C}{\epsilon}
$$

Let us fix the test function $v \in H_{\Gamma_{0}}^{3}(0,1)$ in (3.15) and let $\epsilon$ go to zero. From (3.29), it follows that

$$
\left|\epsilon^{2} \int_{0}^{1} \partial_{2}^{3} \hat{u}^{\epsilon}(\xi)\left(x_{2}\right) \partial_{2}^{3} v\left(x_{2}\right) \mathrm{d} x_{2}\right| \leq C \epsilon .
$$

Moreover, according to (3.26), we deduce that

$$
\begin{array}{r}
\int_{0}^{1}\left(\left(\partial_{2}^{2}-\xi^{2}\right) \hat{u}^{\epsilon}(\xi)\left(x_{2}\right)\left(\partial_{2}^{2}-\xi^{2}\right) v\left(x_{2}\right)+\epsilon^{2}\left(\xi^{6} \hat{u}^{\epsilon}(\xi)\left(x_{2}\right) v\left(x_{2}\right)\right)\right) \mathrm{d} x_{2} \rightarrow \\
\int_{0}^{1}\left(\partial_{2}^{2}-\xi^{2}\right) \hat{u}^{*}(\xi)\left(x_{2}\right)\left(\partial_{2}^{2}-\xi^{2}\right) v\left(x_{2}\right) \mathrm{d} x_{2}
\end{array}
$$


as $\epsilon \rightarrow 0$. Therefore, using the density of the test functions $v$ (note that $H_{\Gamma_{0}}^{3}$ is dense in $H_{\Gamma_{0}}^{2}$, we deduce that $\hat{u}^{*}(\xi)$ is a solution of problem (3.27), hence $\hat{u}^{*}(\xi)=\hat{u}(\xi)$.

Let us now prove that $\hat{u}^{\epsilon}(\xi) \rightarrow \hat{u}(\xi)$ strongly in $H_{\Gamma_{0}}^{2}(0,1)$ as $\epsilon \rightarrow 0$. Recalling the coerciveness character of $\hat{a}$ on $H_{\Gamma_{0}}^{2}(0,1)$, it follows that

$$
C\left\|\hat{u}^{\epsilon}(\xi)-\hat{u}(\xi)\right\|_{H^{2}} \leq \hat{a}\left(\hat{u}^{\epsilon}(\xi)-\hat{u}(\xi), \hat{u}^{\epsilon}(\xi)-\hat{u}(\xi)\right)+\epsilon^{2} \hat{b}\left(\hat{u}^{\epsilon}(\xi), \hat{u}^{\epsilon}(\xi)\right) .
$$

Next, developping the right hand side of (3.32) and using that $\hat{a}\left(\hat{u}^{\epsilon}(\xi), \hat{u}^{\epsilon}(\xi)\right)+$ $\epsilon^{2} \hat{b}\left(\hat{u}^{\epsilon}(\xi), \hat{u}^{\epsilon}(\xi)\right)=\left\langle F, \hat{u}^{\epsilon}(\xi)\right\rangle$ together with $\hat{a}(\hat{u}(\xi), \hat{u}(\xi))=\langle F, \hat{u}(\xi)\rangle$ and $\hat{a}\left(\hat{u}(\xi), \hat{u}^{\epsilon}(\xi)\right)=$ $\hat{a}\left(\hat{u}^{\epsilon}(\xi), \hat{u}(\xi)\right)=\left\langle F, \hat{u}^{\epsilon}(\xi)\right\rangle$, we deduce that (3.32) becomes

$$
C\left\|\hat{u}^{\epsilon}(\xi)-\hat{u}(\xi)\right\|_{H^{2}} \leq\langle F, \hat{u}(\xi)\rangle-\left\langle F, \hat{u}^{\epsilon}(\xi)\right\rangle .
$$

Referring next to the weak convergence of $\hat{u}^{\epsilon}(\xi)$ to $\hat{u}(\xi)$ in $H_{\Gamma_{0}}^{2}(0,1)$, we get the convergence to zero of the right-hand side of (3.33), as $\epsilon$ goes to zero, hence the strong convergence.

We now prove the convergence of $\hat{u}^{\epsilon}$ in the distribution sense with respect to $\xi$. Using the inverse Fourier transform this will give the convergence of $u^{\epsilon}$. Indeed, we shall prove the following theorem:

Theorem 3.4. Let $\hat{u}^{\epsilon}$ and $\hat{u}$ be the solutions of (3.15) and (2.7) respectively. The following convergence holds, as $\epsilon$ goes to zero:

$$
\hat{u}^{\epsilon} \rightarrow \hat{u} \text { in } D^{\prime}\left(\mathbb{R}_{\xi} ; H_{\Gamma_{0}}^{2}(0,1)\right) \text {. }
$$

Moreover, for fixed $x_{2}$, we have:

$$
\begin{aligned}
& \hat{u}^{\epsilon}\left(., x_{2}\right) \rightarrow \hat{u}\left(., x_{2}\right) \text { in } D^{\prime}\left(\mathbb{R}_{\xi}\right), \\
& u^{\epsilon}\left(., x_{2}\right) \rightarrow u\left(., x_{2}\right) \text { in } Z^{\prime}\left(\mathbb{R}_{x_{1}}\right) .
\end{aligned}
$$

where $u$ and $u^{\varepsilon}$ are the solutions of (1.1) and (3.1) respectively, with the loading (2.6).

To do so, we will use the two following lemmas:

Lemma 3.5. Let $I_{\xi}$ be a bounded interval of $\mathbb{R}_{\xi}$, then the sequence $\hat{u}^{\epsilon}(\xi)$ of solutions of (3.15) is such that

$$
\hat{u}^{\epsilon} \rightarrow \hat{u}^{*} \text { weakly in } L^{2}\left(I_{\xi} ; H_{\Gamma_{0}}^{2}(0,1)\right) \text { as } \epsilon \rightarrow 0 .
$$

for some $\hat{u}^{*} \in L^{2}\left(I_{\xi} ; H_{\Gamma_{0}}^{2}(0,1)\right)$. Moreover, the trace of $\hat{u}^{\epsilon}$ over $x_{2}=$ const weakly converges in $L^{2}\left(I_{\xi}\right)$ to the trace of $\hat{u}^{*}$. 
Proof of Lemma 3.5. Recalling that the coerciveness constant in Lemma 3.1 is uniform with respect to $\xi \in I_{\xi}$, we deduce that the a priori estimate of $\hat{u}^{\epsilon}(\xi)$ in $H_{\Gamma_{0}}^{2}(0,1)$ is also uniform. Therefore, $\hat{u}^{\epsilon}$ remains bounded in the space $L^{\infty}\left(I_{\xi} ; H_{\Gamma_{0}}^{2}(0,1)\right)$, hence in the Hilbert space $L^{2}\left(I_{\xi} ; H_{\Gamma_{0}}^{2}(0,1)\right)$. Now, similarly to the proof of Lemma 3.3, we deduce that there exists $\hat{u}^{*} \in L^{2}\left(I_{\xi} ; H_{\Gamma_{0}}^{2}(0,1)\right)$ such that

$$
\hat{u}^{\epsilon} \rightarrow \hat{u}^{*} \text { weakly in } L^{2}\left(I_{\xi} ; H_{\Gamma_{0}}^{2}(0,1)\right) \text { as } \epsilon \rightarrow 0,
$$

for some $\hat{u}^{*}$ in $L^{2}\left(I_{\xi} ; H_{\Gamma_{0}}^{2}(0,1)\right)$. The convergence of the traces follows from the trace theorem in $H^{2}(0,1)$.

Lemma 3.6. Under the hypotheses of Lemma 3.3, we have:

$$
\hat{u}^{\epsilon} \rightarrow \hat{u} \text { in } D^{\prime}\left(\mathbb{R}_{\xi} ; H_{\Gamma_{0}}^{2}(0,1)\right) \text { as } \epsilon \rightarrow 0,
$$

where $\hat{u}$ is the solution of (2.7). Moreover, the trace of $\hat{u}^{\epsilon}$ over $x_{2}=$ const converges in $D^{\prime}$ to the trace of $\hat{u}$.

Proof of Lemma 3.6. Let $\theta \in \mathcal{D}\left(\mathbb{R}_{\xi}\right)$ be a test function of distributions. Let $I_{\xi}$ be a bounded interval of $\mathbb{R}_{\xi}$ containing the support of $\theta$. Let us first consider a fixed test function $v$ in (3.15). Next, multiplying by $\theta(\epsilon)$ and integrating (3.15) in $\xi$, we get

$$
\begin{aligned}
& \int_{I_{\xi}} \int_{0}^{1}\left(\left(\partial_{2}^{2}-\xi^{2}\right) \hat{u}^{\epsilon}(\xi)\left(x_{2}\right)\left(\partial_{2}^{2}-\xi^{2}\right) v\left(x_{2}\right)+\right. \\
& \left.\epsilon^{2}\left(\xi^{6} \hat{u}^{\epsilon}(\xi)\left(x_{2}\right) v\left(x_{2}\right)+\partial_{2}^{3} \hat{u}^{\epsilon}(\xi)\left(x_{2}\right) \partial_{2}^{3} v\left(x_{2}\right)\right)\right) \theta(\xi) \mathrm{d} x_{2} \mathrm{~d} \xi \\
= & \int_{I_{\xi}} \int_{0}^{1} F\left(x_{2}\right) v\left(x_{2}\right) \theta(\xi) \mathrm{d} x_{2} \mathrm{~d} \xi .
\end{aligned}
$$

Passing to the limit in (3.40) according to Lemma 3.3, (3.30), this gives

$$
\int_{I_{\xi}} \int_{0}^{1}\left(\partial_{2}^{2}-\xi^{2}\right) \hat{u}(\xi)\left(x_{2}\right)\left(\partial_{2}^{2}-\xi^{2}\right) v\left(x_{2}\right) \theta(\xi) \mathrm{d} x_{2} \mathrm{~d} \xi=\int_{I_{\xi}} \int_{0}^{1} F\left(x_{2}\right) v\left(x_{2}\right) \theta(\xi) \mathrm{d} x_{2} \mathrm{~d} \xi .
$$

Therefore, we deduce that

$$
\int_{0}^{1}\left(\partial_{2}^{2}-\xi^{2}\right) \hat{u}(\xi)\left(x_{2}\right)\left(\partial_{2}^{2}-\xi^{2}\right) v\left(x_{2}\right) \mathrm{d} x_{2}=\int_{0}^{1} F\left(x_{2}\right) v\left(x_{2}\right) \mathrm{d} x_{2},
$$

in the sense of distributions of $\xi$. The convergence of the traces follows from the trace theorem in $H^{2}(0,1)$. 
The proof of theorem 3.4 is then obvious. The convergence of the Fourier transforms and of the corresponding traces on $x_{2}=$ const is merely Lemma 3.6. Then, we use the fact that the Fourier transform (in fact inverse Fourier transform) defines an isomorphism from $D^{\prime}\left(\mathbb{R}_{\xi}\right)$ on $Z^{\prime}\left(\mathbb{R}_{x}\right)$.

The previous Theorem 3.4 is our main result on the convergence $\varepsilon \rightarrow 0$. Obviously, it is much more explicit in the description of the convergence of the Fourier transforms than in the $u^{\varepsilon}$ themselves. This is natural, as the existence (in the sense of functions of $x_{2}$ with values in $Z^{\prime}\left(\mathbb{R}_{x_{1}}\right)$ ) was also obtained by Fourier transform. Likely, the convergence in Theorem 3.4 cannot be very much improved.

It will prove useful to consider the asymptotic behavior of $\hat{u}^{\varepsilon}(\xi)$ with fixed $\varepsilon>0$ when $|\xi| \rightarrow \infty$. Under these hypotheses, by inspection of (3.15) on account of (3.10) and (3.11) it appears that the main term is $\varepsilon^{2} \xi^{6} \hat{u}^{\varepsilon}(\xi)=F$ so that $\hat{u}^{\varepsilon}(\xi)$ should behave as $\varepsilon^{-2} \xi^{-6} F$ for $|\xi| \rightarrow \infty$. A rigorous statement of this property is given by the next theorem. Its proof is a mere adaptation of classical singular perturbation theory, close in particular to Lemma 3.3.

Theorem 3.7. Let $\epsilon>0$ be given and let $\hat{u}^{\epsilon}(\xi)$ be a solution of (3.15). Let us set $\hat{v}^{\epsilon}(\xi)=\epsilon^{2} \xi^{6} \hat{u}^{\epsilon}(\xi)$, then

$$
\hat{v}^{\epsilon}(\xi) \rightarrow F \text { strongly in } L^{2}(0,1) \text {, as }|\xi| \rightarrow \infty .
$$

Proof. Let $\epsilon>0$ be given. First, we prove the weak convergence. Recalling that $\hat{u}^{\epsilon}(\xi)$ is a solution of (3.15) and taking $\hat{v}^{\epsilon}(\xi)=\epsilon^{2} \xi^{6} \hat{u}^{\epsilon}(\xi)$ as a test function in (3.15), we obtain

$$
\epsilon^{-2} \xi^{-6}\left\|\left(\partial_{2}^{2}-\xi^{2}\right) \hat{v}^{\epsilon}(\xi)\right\|_{L^{2}}^{2}+\left\|\hat{v}^{\epsilon}(\xi)\right\|_{L^{2}}^{2}+\xi^{-6}\left\|\partial_{2}^{3} \hat{v}^{\epsilon}(\xi)\right\|_{L^{2}}^{2}=\left\langle F, \hat{v}^{\epsilon}(\xi)\right\rangle .
$$

Furthermore, since $\hat{u}^{\epsilon}(\xi)$ belongs to $H_{\Gamma_{0}}^{3}(0,1)$, using three times Poincaré's inequality together with Cauchy Schwartz and remembering that $F$ belongs to $L^{2}(0,1)$, this yields:

$$
\begin{aligned}
\left\|\hat{v}^{\epsilon}(\xi)\right\|_{L^{2}}^{2} & \left.\leq \epsilon^{-2} \xi^{-6}\left\|\left(\partial_{2}^{2}-\xi^{2}\right) \hat{v}^{\epsilon}(\xi)\right\|_{L^{2}}^{2}+\left\|\hat{v}^{\epsilon}(\xi)\right\|_{L^{2}}^{2}+\xi^{-6}\left\|\hat{v}^{\epsilon}(\xi)\right\|_{H^{3}}^{2} .44\right) \\
& \leq C\left\|\hat{v}^{\epsilon}(\xi)\right\|_{L^{2}} .
\end{aligned}
$$

Consequently, there exists $\hat{v}^{\epsilon *}$ in $L^{2}(0,1)$ such that for a subsequence, not relabelled, $\hat{v}^{\epsilon}(\xi) \rightarrow \hat{v}^{\epsilon *}(\xi)$ weakly in $L^{2}(0,1)$, as $|\xi| \rightarrow \infty$.

Next, we prove that $\hat{v}^{\epsilon *}=F$. $>$ From (3.44) again, we deduce that

$$
\epsilon^{-2} \xi^{-2}\left\|\left(\xi^{-2} \partial_{2}^{2}-1\right) \hat{v}^{\epsilon}(\xi)\right\|_{L^{2}}^{2} \leq C \text { and } \xi^{-6}\left\|\hat{v}^{\epsilon}(\xi)\right\|_{H^{3}}^{2} \leq C .
$$

Let us fix the test function $v \in H_{\Gamma_{0}}^{3}(0,1)$ in (3.15), from (3.45) it follows that

$$
\xi^{-6}\left|\int_{0}^{1} \partial_{2}^{3} \hat{v}^{\epsilon}(\xi)\left(x_{2}\right) \partial_{2}^{3} v\left(x_{2}\right) \mathrm{d} x_{2}\right| \leq \frac{C}{|\xi|^{3}} \rightarrow 0, \text { as }|\xi| \rightarrow \infty .
$$

Similarly, we have

$$
\epsilon^{-2} \xi^{-2}\left|\int_{0}^{1}\left(\xi^{-2} \partial_{2}^{2}-1\right) \hat{v}^{\epsilon}(\xi)\left(x_{2}\right)\left(\xi^{-2} \partial_{2}^{2}-1\right) v\left(x_{2}\right) \mathrm{d} x_{2}\right| \leq \frac{C}{\epsilon|\xi|} \rightarrow 0
$$


as $|\xi| \rightarrow \infty$. Since, for all $\epsilon>0$, fixed, we have $\left\langle F, \hat{v}^{\epsilon}\right\rangle \rightarrow\left\langle F, \hat{v}^{\epsilon *}\right\rangle$, as $|\xi| \rightarrow \infty$, we deduce that for all $\epsilon>0, \hat{v}^{\epsilon *}=F$.

Finally using that

$$
\begin{aligned}
\epsilon^{-2} \xi^{-6}\left\|\left(\partial_{2}^{2}-\xi^{2}\right) \hat{v}^{\epsilon}(\xi)\right\|_{L^{2}}^{2} & +\left\|\hat{v}^{\epsilon}(\xi)-F\right\|_{L^{2}}^{2}+\xi^{-6}\left\|\partial_{2}^{3} \hat{v}^{\epsilon}(\xi)\right\|_{L^{2}}^{2} \\
& =\left\langle F, \hat{v}^{\epsilon}(\xi)\right\rangle+\|F\|_{L^{2}}^{2}-2\left\langle F, \hat{v}^{\epsilon}(\xi)\right\rangle
\end{aligned}
$$

and recalling the weak convergences, we deduce the strong convergence.

\section{Emergence of a new small parameter in the previous problem and formal asymptotics}

This section has a formal character. Its goal is to get an easily understandable description of $\hat{u}^{\varepsilon}(\xi)$ with small $\varepsilon$.

For obvious reasons, the limit properties of $u^{\varepsilon}$ when $\varepsilon \rightarrow 0$ are more clear when considered in terms of the Fourier transform $\hat{u}^{\varepsilon}(\xi)$. The limit $\hat{u}(\xi)$ behaves for $|\xi| \rightarrow \infty$ as indicated in (2.19) or (2.21), i. e. with exponential growing. On the other hand, according to Theorem $3.2, \hat{u}^{\varepsilon}\left(\xi, x_{2}\right)$ with fixed small $\varepsilon$ behaves when $|\xi| \rightarrow \infty$ in the form:

$$
\hat{u}^{\epsilon}(\xi) \approx \epsilon^{-2} \xi^{-6} F .
$$

From the previous considerations it is obvious that $\hat{u}^{\varepsilon}(\xi)$ converges to $\hat{u}(\xi)$ as $\varepsilon \rightarrow 0$ in various topologies (see Lemma 3.3 and Theorem 3.1) but certainly not uniformly in $\xi \in \mathbb{R}$. Clearly, $\hat{u}^{\varepsilon}(\xi)$ is close to $\hat{u}(\xi)$ unless for very large $|\xi|$, where it is approximated by (4.1). Referring to (3.15) with (3.10) and (3.11) it means that an approximation of the solution is obtained neglecting the perturbation term (i. e., taking $\varepsilon=0$ ) unless for large values of $|\xi|$, for which only the leading term $\varepsilon^{2} \xi^{6} \hat{u}^{\varepsilon}(\xi)$ should be retained. Therefore, the exact expression of $\hat{u}^{\epsilon}(\xi)$ is a smooth function matching these two parts for finite and large $|\xi|$. Likely, the first approximation is also valid for moderately large values of $|\xi|$, so that we will have:

$$
\hat{u}^{\epsilon}\left(\xi, x_{2}\right) \approx K e^{\left(c+x_{2}\right)|\xi|}
$$

or rather $\left|\hat{u}^{\epsilon}\left(\xi, x_{2}\right)\right|$ is comprised between the expression at the right hand side for various values of $c$, but we shall see that the result is independent of them. In order to define a transition region between the two previous patterns, where we can neglect none of these two parts with respect to the other, we must have

$$
e^{\left(c+x_{2}\right)|\xi|}=\mathcal{O}\left(\epsilon^{-2}\right) .
$$

Consequently, the characteristic frequency is of order:

$$
|\xi|=\mathcal{O}(|\log \epsilon|)
$$


In other words, (4.4) defines the transition region between the two regions with "fixed $\xi$ " and "large $\xi$ ". This transition region will play an important role in the sequel. It is characterized by the frequencies $\mathcal{O}(|\log \varepsilon|)$; according to the growing properties of the function $\log$, when $\varepsilon$ is small, they are "very moderately large". Equivalently, the corresponding wave lengths in $x_{1}$ are "very moderately small", of order $\mathcal{O}\left(|\log \varepsilon|^{-1}\right)$. Moreover, the values of the functions in that region are very large for small $\varepsilon$, of order close to $0\left(\varepsilon^{-2}\right)$.

Let us consider $\hat{u}^{\epsilon}(\xi)$ with a fixed, small value of $\varepsilon$. A good approximation of it is given by $\hat{u}(\xi)$ and $\epsilon^{-2} \xi^{-6} F$ in the regions $|\xi|<\mathcal{O}(|\log \varepsilon|)$ and $|\xi|>\mathcal{O}(|\log \varepsilon|)$ respectively, whereas in the intermediate region the totality of the terms in (3.15) should be taken into account. An obvious inspection of the graphs of the functions in (4.1) and (4.2) show that the transition region should bear the most quantitatively important part of $\hat{u}^{\epsilon}(\xi)$; we shall see later numerical examples of that fact.

We are now giving an heuristic approximate analysis of $\hat{u}^{\epsilon}(\xi)$ for small $\varepsilon$ and "moderately large" $|\xi|$. According to the previous considerations, it will be a good quantitative approximation of $u^{\varepsilon}$, but we shall comment this point a little later (see Remark 16)

Let us consider again the problem (3.15) when $\varepsilon$ and $\xi$ are considered as parameters, with $\varepsilon \rightarrow 0$ and $|\xi|=\mathcal{O}(|\log \epsilon|)$. In order to allow explicit computations, we shall take

$$
F\left(x_{2}\right)=\delta\left(x_{2}-c\right) .
$$

This $F$ is not in $L^{2}(0,1)$ and then out of the framework of the previous considerations; but this point is not essential (see later Remark 15)

Referring to (3.15) with the $F$ specified in (4.5), the problem amounts to the minimization of the functional

$$
\hat{a}(v, v)+\varepsilon^{2} \hat{b}(v, v)-2 v(c)
$$

in the space $H_{\Gamma_{0}}^{3}(0,1)$. We shall now use general features of minimization of functionals depending on parameters under various hypotheses (they are more or less intuitive, but we may refer to [16] in this connection). It should be recalled that the forms $\hat{a}$ and $\hat{b}$ depend on $\xi$, but, according to the relative values of $\varepsilon$ and $\xi$ in the considered region, we may practically consider that $\xi$ is "large but fixed" and $\varepsilon$ tends to 0 in order to find a formal asymptotics. Clearly, the natural trend of the solution of the minimization problem is to avoid the term $\hat{a}(v, v)$ which is "expensive" in energy, with respect to the other, which bears the small factor $\varepsilon^{2}$. According to (3.10), the vanishing of the form $\hat{a}$ amounts to $\left(\partial_{2}^{2}-|\xi|^{2}\right) v=0$, i. e. $v$ is in the two - dimensional space

$$
v=\alpha e^{|\xi| x_{2}}+\beta e^{-|\xi| x_{2}} .
$$

on the whole interval $x_{2} \in(0,1)$. It then appears that, when imposing the (essential) boundary conditions on $x_{2}=0$ the subspace reduces to the 0 vector, so that this first idea is too coarse for describing the asymptotics. 
We are then enlarging that subspace. To this end, we know that the (exact) solution satisfies the homogeneous equation

$$
\left(\left(\partial_{2}^{2}-\xi^{2}\right)\left(\partial_{2}^{2}-\xi^{2}\right)-\epsilon^{2}\left(\xi^{6}+\partial_{2}^{6}\right)\right) \hat{u}^{\epsilon}\left(\xi, x_{2}\right)=0
$$

on each of the intervals $(0, c)$ and $(c, 1)$. Then, on each one of these intervals, it is a linear combination of the six functions $e^{\lambda_{i} x_{2}}$ where $\lambda_{i}$ are the roots of the equation:

$$
\left(\lambda^{2}-\xi^{2}\right)^{2}-\epsilon^{2}\left(\xi^{6}+\lambda^{6}\right)=0 .
$$

We are now solving approximatively this equation recalling that $\varepsilon$ is small and $|\xi|$ moderately large. It immediately appears that there are two roots close to $|\xi|$, two roots close to $-|\xi|$, and two roots with very large modulus, approximatively equal to $1 / \varepsilon$ and $-1 / \varepsilon$. The two first assertions follow directly from (4.9) with $\varepsilon=0$, whereas the last follows from the change of unknown $\lambda=\mu / \varepsilon$, which gives

$$
\left(\mu^{2}-\varepsilon^{2} \xi^{2}\right)^{2}-\epsilon^{6} \xi^{6}+\mu^{6}=0
$$

and then taking $\varepsilon=0$. Going on with our approximation, we may consider (see for instance [20] for details) that the two roots close to $|\xi|$ are in fact a double root, as well as the two close to $-|\xi|$. It means that, on each of the intervals $(0, c)$ and $(c, 1)$ we may consider, in addition to (4.7), functions of the form:

$$
\gamma x_{2} e^{|\xi| x_{2}}+\delta x_{2} e^{-|\xi| x_{2}}+\zeta e^{\frac{-1}{\epsilon} x_{2}}+\theta e^{\frac{1}{\epsilon} x_{2}} .
$$

Moreover, in the framework of our approximation, we observe that, as $|\xi|$ is large and $\varepsilon$ small, the functions with coefficients $\gamma$ and $\theta$ bear a large amount of energy associated with the form $\hat{a}$, and should be disregarded. As a result, at the present state, on each of the intervals $(0, c)$ and $(c, 1)$ we may consider, in addition to (4.7), functions of the form:

$$
\delta x_{2} e^{-|\xi| x_{2}}+\zeta e^{\frac{-1}{\epsilon} x_{2}} .
$$

But, as the functions must be in the space $H_{\Gamma_{0}}^{3}(0,1)$, the traces of the functions and of the first and second order derivatives must be the same on both sides of $x_{2}=$ $c$ (obviously this property is concerned with the space of minimization, and has nothing to do with the natural boundary conditions (3.14) which are not concerned by the space of minimization). As these three conditions are automatically satisfied by (4.7), which is valid on the whole interval, we only must prescribe them on (4.12). This evidently shows that $\delta$ and $\theta$ should take the same value on both intervals. This gives, on the whole interval, $(0,1)$, functions of the form

$$
v=\alpha e^{|\xi| x_{2}}+\beta e^{-|\xi| x_{2}}+\delta x_{2} e^{-|\xi| x_{2}}+\zeta e^{\frac{-1}{\epsilon} x_{2}} .
$$

We now have at our disposal a four - dimensional space (instead of the two dimensional one (4.7)) and prescribing the (essential) boundary conditions (3.13) on $x_{2}=0$ we get: 


$$
\left\{\begin{aligned}
\delta & =\frac{1}{2 \xi}\left(\frac{1}{\epsilon^{2}}-\xi^{2}\right) \zeta \\
\beta & =\frac{1}{4 \xi^{2}}\left(\frac{1}{\epsilon}-3|\xi|\right)\left(\frac{1}{\epsilon}+|\xi|\right) \zeta \\
\alpha & =-\frac{1}{4 \xi^{2}}\left(\frac{1}{\epsilon}-|\xi|\right)^{2} \zeta .
\end{aligned}\right.
$$

and the space of minimization becomes the one - dimensional space

$$
v\left(\xi, x_{2}\right)=A(\xi, \varepsilon) w^{\varepsilon}\left(\xi, x_{2}\right)
$$

with

$w^{\varepsilon}\left(\xi, x_{2}\right)=\left((1-\epsilon \xi)^{2} \frac{\sinh \left(|\xi| x_{2}\right)}{|\xi|}-\left(1-\epsilon^{2} \xi^{2}\right) x_{2} e^{-|\xi| x_{2}}-2 \epsilon^{2}|\xi|\left(e^{-\frac{1}{\epsilon} x_{2}}-e^{-|\xi| x_{2}}\right)\right)$.

We note that, within our approximation, as $\varepsilon$ is small, as well as $\varepsilon|\xi|$, we may also consider

$$
w^{\varepsilon}\left(\xi, x_{2}\right)=\left(\frac{\sinh \left(|\xi| x_{2}\right)}{|\xi|}-x_{2} e^{-|\xi| x_{2}}-2 \epsilon^{2}|\xi| e^{-\frac{1}{\epsilon} x_{2}}\right)
$$

where it should be noted that the last term is small with respect to the others, so that it should also be discarded; we only keep it in order to show that the boundary conditions at $x_{2}=0$ are (approximatively) satisfied; in fact, that term is a narrow boundary layer near $x_{2}=0$, but it will not play any role in the sequel.

Remark 12. It should be noticed that this result (which is the main one of our formal asymptotics) is independent of the point $c$ of application of the point loading. Accordingly, it may be used for general loadings, which may be obtained by integration of elementary loadings with variable $c \in(0,1)$.

The approximate solution of the minimization problem is now immediate as it is reduced to the one - dimensional space (4.15). Writing

$$
\hat{u}^{\varepsilon}\left(\xi, x_{2}\right)=A(\xi, \varepsilon) w^{\varepsilon}\left(\xi, x_{2}\right),
$$

and recalling that $\hat{u}^{\epsilon}\left(\xi, x_{2}\right)$ is the solution of the variational problem given by (3.15), we deduce that

$$
\hat{a}\left(\hat{u}^{\epsilon}(\xi), \hat{u}^{\epsilon}(\xi)\right)+\epsilon^{2} \hat{b}\left(\hat{u}^{\epsilon}(\xi), \hat{u}^{\epsilon}(\xi)\right)=\left\langle\hat{u}^{\epsilon}(\xi), \delta\left(x_{2}-c\right)\right\rangle,
$$

so that

$$
A(\xi, \epsilon)^{2}\left(\hat{a}\left(w^{\epsilon}(\xi), w^{\epsilon}(\xi)\right)+\epsilon^{2} \hat{b}\left(w^{\epsilon}(\xi), w^{\epsilon}(\xi)\right)\right)=A(\xi, \epsilon)\left\langle w^{\epsilon}(\xi), \delta\left(x_{2}-c\right)\right\rangle
$$

and then

$$
A(\xi, \epsilon)=\frac{w^{\epsilon}(\xi, c)}{\hat{a}\left(w^{\epsilon}(\xi), w^{\epsilon}(\xi)\right)+\epsilon^{2} \hat{b}\left(w^{\epsilon}(\xi), w^{\epsilon}(\xi)\right)}
$$


Furthermore, thanks to the fact that $\epsilon|\xi|<<1$, the approximate expressions of $w^{\epsilon}(\xi), \hat{a}\left(w^{\epsilon}(\xi), w^{\epsilon}(\xi)\right)$ and $\hat{b}\left(w^{\epsilon}(\xi), w^{\epsilon}(\xi)\right)$ are (recall (4.17) and the comments after it):

$$
\begin{gathered}
w^{\epsilon}\left(\xi, x_{2}\right) \approx \\
w_{\mathrm{app}}\left(\xi, x_{2}\right)=\frac{\sinh \left(|\xi| x_{2}\right)}{|\xi|}-x_{2} e^{-|\xi| x_{2}} \\
\hat{a}\left(w^{\epsilon}(\xi), w^{\epsilon}(\xi)\right) \approx 2|\xi| \\
\hat{b}\left(w^{\epsilon}(\xi), w^{\epsilon}(\xi)\right) \approx \frac{|\xi|^{3}}{4} e^{2|\xi|}
\end{gathered}
$$

hence,

$$
\hat{a}\left(w^{\epsilon}(\xi), w^{\epsilon}(\xi)\right)+\epsilon^{2} \hat{b}\left(w^{\epsilon}(\xi), w^{\epsilon}(\xi)\right) \approx 2|\xi|+\epsilon^{2} \frac{|\xi|^{3}}{4} e^{2|\xi|} .
$$

Then (4.20) becomes

$$
A(\xi, \epsilon)=\frac{w^{\epsilon}(\xi, c)}{2|\xi|+\epsilon^{2} \frac{\xi^{3}}{4} e^{2|\xi|}} .
$$

and the final expression of the approximate solution is

$$
\hat{u}^{\varepsilon}\left(\xi, x_{2}\right)=\frac{w^{\epsilon}(\xi, c) w^{\epsilon}\left(\xi, x_{2}\right)}{2|\xi|+\epsilon^{2} \frac{|\xi|^{3}}{4} e^{2|\xi|}} .
$$

where $w^{\varepsilon}$ is given by (4.16) or even by its approximate expression (4.21).

Recalling (4.18), (4.20), (4.21) together with (4.24), we obtain that the total energy is

$$
\begin{aligned}
\hat{a}\left(\hat{u}^{\epsilon}(\xi), \hat{u}^{\epsilon}(\xi)\right)+\epsilon^{2} \hat{b}\left(\hat{u}^{\epsilon}(\xi), \hat{u}^{\epsilon}(\xi)\right) & =A(\xi, \epsilon)^{2}\left(\hat{a}\left(w^{\epsilon}(\xi), w^{\epsilon}(\xi)\right)+\epsilon^{2} \hat{b}\left(w^{\epsilon}(\xi), w^{\epsilon}(\xi)\right)\right) \\
& \approx \frac{e^{2|\xi| c}}{|\xi|^{2}\left(8|\xi|+\epsilon^{2}|\xi|^{3} e^{2|\xi|}\right)}
\end{aligned}
$$

Remark 13. When computing $\hat{a}\left(w^{\epsilon}(\xi), w^{\epsilon}(\xi)\right)=\int_{0}^{1}\left|\left(\partial_{2}^{2}-\xi^{2}\right) w^{\epsilon}(\xi)\left(x_{2}\right)\right|^{2} \mathrm{~d} x_{2}$, with (4.17), i. e.

$$
\begin{array}{r}
\int_{0}^{1}\left(-2|\xi| e^{-|\xi| x_{2}}+2|\xi| e^{-\frac{1}{\epsilon} x_{2}}\left(1-\epsilon^{2} \xi^{2}\right)\right)^{2} \mathrm{~d} x_{2}= \\
-2|\xi|\left(e^{-|\xi|}-1\right)+8 \epsilon|\xi|(1-\epsilon|\xi|)\left(e^{-\frac{1}{\epsilon}-|\xi|}-1\right)-2 \epsilon|\xi|^{2}\left(1-\epsilon^{2} \xi^{2}\right)\left(e^{-\frac{2}{\epsilon}}-1\right),
\end{array}
$$

it is easily seen that the previous energy localizes in the vicinity of $x_{2}=0$ when $|\xi|$ tends to infinity, since $\epsilon|\xi|<<1$. It means that, taking any $\gamma$ in $(0,1)$,

$$
\begin{array}{r}
\int_{0}^{\gamma}\left|\left(\partial_{2}^{2}-\xi^{2}\right) w^{\epsilon}(\xi)\left(x_{2}\right)\right|^{2} \mathrm{~d} x_{2}= \\
-2|\xi|\left(e^{-|\xi| \gamma}-1\right)+8 \epsilon|\xi|(1-\epsilon|\xi|)\left(e^{-\left(\frac{1}{\epsilon}+|\xi|\right) \gamma}-1\right)-2 \epsilon|\xi|^{2}\left(1-\epsilon^{2} \xi^{2}\right)\left(e^{-\frac{2 \gamma}{\epsilon}}-1\right)
\end{array}
$$


is asymptotically equal to $\hat{a}\left(w^{\epsilon}(\xi), w^{\epsilon}(\xi)\right)$ for $|\xi| \rightarrow+\infty$. This result is also evident form the fact that the leading part of the $\hat{a}\left(w^{\epsilon}(\xi), w^{\epsilon}(\xi)\right)$ energy depends on the second term in the expression (4.17).

Oppositely, the $\hat{b}$ energy depends mainly on the first term of the expression (4.17) and the $\hat{b}$ energy localizes in the vicinity of $x_{2}=1$.

This is the phenomenon of "migration of energies" in the sense that as $|\xi|$ tends to infinity, the $\hat{a}$ and $\hat{b}$ energies migrate towards $\Gamma_{0}$ and $\Gamma_{1}$ respectively, whereas the open interval $(0,1)$ tends to become free of energy.

Remark 14. Let us denote in this remark $\hat{u}_{c}^{\epsilon}$ and $\hat{u}_{c^{\prime}}^{\epsilon}$ the solutions associated to loads located in $c$ and $c^{\prime}$. Recalling that, within our formal asymptotics $\hat{u}_{c}^{\epsilon}\left(\xi, x_{2}\right)=$ $A_{c}(\xi, \epsilon) w^{\epsilon}\left(\xi, x_{2}\right)$ with $A_{c}(\xi, \epsilon)=\frac{w^{\epsilon}(\xi, c)}{\hat{a}\left(w^{\epsilon}(\xi), w^{\epsilon}(\xi)\right)+\epsilon^{2} \hat{b}\left(w^{\epsilon}(\xi), w^{\epsilon}(\xi)\right)}$, we deduce the reciprocity property

$$
\hat{u}_{c}^{\epsilon}\left(\xi, c^{\prime}\right)=\hat{u}_{c^{\prime}}^{\epsilon}(\xi, c) \text {. }
$$

Remark 15. According to Remark 12, in the case when the loading is $f\left(x_{1}, x_{2}\right)=$ $\delta\left(x_{1}\right) F\left(x_{2}\right)$ with $F \in L^{2}(0,1)$, equations (4.19) and (4.20) become

$$
A(\xi, \epsilon)^{2}\left(\hat{a}\left(w^{\epsilon}(\xi), w^{\epsilon}(\xi)\right)+\epsilon^{2} \hat{b}\left(w^{\epsilon}(\xi), w^{\epsilon}(\xi)\right)\right)=A(\xi, \epsilon)\left\langle F, \hat{w}^{\epsilon}(\xi)\right\rangle
$$

and

$$
A(\xi, \epsilon)=\frac{\int_{0}^{1} F\left(x_{2}\right) w^{\epsilon}\left(\xi, x_{2}\right) \mathrm{d} x_{2}}{2|\xi|+\epsilon^{2} \frac{\left.\left.\right|^{\mid}\right|^{3}}{4} e^{2|\xi|}} .
$$

respectively

Remark 16. The previous formal asymptotics was developed for "small" $\epsilon$ and "moderately large " $|\xi|$, where $u^{\epsilon}$ takes large values (nearby $\mathcal{O}\left(\epsilon^{-2}\right)$ according to the considerations after (4.4)). Nevertheless, we shall apply this asymptotics for small $\epsilon$ and any $\xi \in \mathbb{R}$ (in other words, the expression (4.26) and analogous ones will be used for small $\epsilon$ and any $\xi$ ). This is justified by the fact that $\hat{u}^{\epsilon}$ as well as its approximated values are of order $\mathcal{O}(1)$ for $\xi=\mathcal{O}(1)$ and vanishing small for $\xi \rightarrow \infty$.

We carried out a few numerical experiments on the approximated $\hat{u}^{\epsilon}(\xi, 1)$ and $u^{\epsilon}\left(x_{1}, 1\right)$ using the free scientific computing package scilab (http://www.scilab.org)

We include a sequence of four graphics. Since the functions we are studying are real and even, the fourier transform is also real and even, and we only represent the functions for positive $x$ (or $\xi$ ) values.

The first series of numerical experiments, (Figures 1,2,3) corresponds to the loading $f\left(x_{1}, x_{2}\right)=\delta(x 1) \delta\left(x_{2}-1\right)$ (note that in that case the force is applied on the boundary $\Gamma_{1}$ and in fact it appears as a non homogeneous boundary condition). On Fig 1 we represent $\hat{u}^{\epsilon}(\xi, 1)$ on the left and $u^{\epsilon}\left(x_{1}, 1\right)$ on the right for three values of $\epsilon: \epsilon=10^{-3}, \epsilon=10^{-4}$ and $\epsilon=10^{-5}$. In the left graphics, the abscisse-axis is $\xi \in[0,32]$ while in the right one, it is $x_{1} \in[0,0.8]$. We observe that both $\left|\hat{u}^{\epsilon}(\xi, 1)\right|$ and $\left|u^{\epsilon}\left(x_{1}, 1\right)\right|$ (indeed we can not see $u^{10^{-3}}$ since it is too small in compraison with the two others) increase drastically as $\epsilon$ decreases. This is in good agreement 

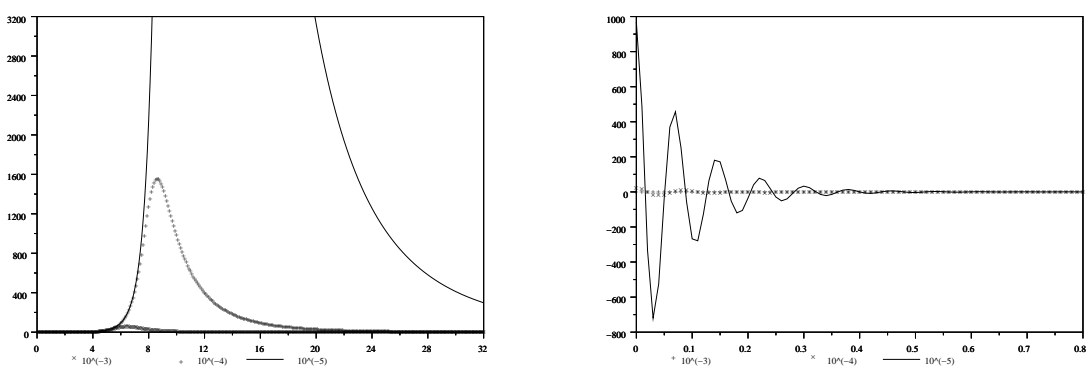

Figure 1: $\hat{u}^{\epsilon}(\xi, 1)$ on the left and $u^{\epsilon}\left(x_{1}, 1\right)$ on the right, for $\epsilon=10^{-3}, 10^{-4}$ and $10^{-5}$

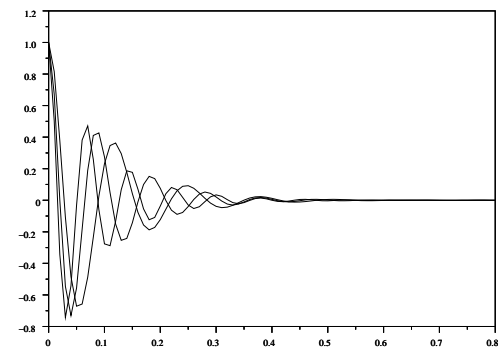

Figure 2: $u^{\epsilon}\left(x_{1}, 1\right) /\left|u^{\epsilon}(0,1)\right|$ for $\epsilon=10^{-3}, 10^{-4}$ and $10^{-5}$

with the fact that neither the limit of $\hat{u}^{\epsilon}(\xi, 1)$ nor the limit of $u^{\epsilon}\left(x_{1}, 1\right)$, as $\epsilon$ tends to zero, belong to the space $S^{\prime}$.

In the second graphic, Figure 2, we represent $u^{\epsilon}\left(x_{1}, 1\right) /\left|u^{\epsilon}(0,1)\right|$, for the same three values of $\epsilon: \epsilon=10^{-3}, \epsilon=10^{-4}$ and $\epsilon=10^{-5}$. The abscisse-axis is $x_{1} \in[0,0.8]$. We observe that the shapes of solutions become more and more complex as $\varepsilon$ decreases. But this process is very slow, in fact it is associated with the new parameter $|\log \varepsilon|$, which increases very slowly as $\varepsilon$ decreases

In order to observe this complexification phenomenon, let us define the essential domain as the set of all $x_{1}$ such that $u^{\epsilon}\left(x_{1}, 1\right) /\left|u^{\epsilon}(0,1)\right| \geq 10^{-2}$. On the essential domain, we will count the number of maxima of the curves corresponding to different values of $\epsilon$. If this number is increasing as $\epsilon$ decreases, we shall say that there is complexification. This is more apparent in Figure 3, where we represented $u^{\epsilon}\left(x_{1}, 1\right) /\left|u^{\epsilon}(0,1)\right|$ for two values of $\epsilon: \epsilon=10^{-3}$ on the left and $\epsilon=10^{-5}$ on the right. For $\epsilon=10^{-5}$, we can see four maxima while for $\epsilon=10^{-3}$ we only observe three maxima. As we see, this complexification process takes place very slowly as $\varepsilon$ decreases. This is not very surprising since it is associated with the new parameter $|\log \varepsilon|$, which increases very slowly as $\varepsilon$ decreases. 

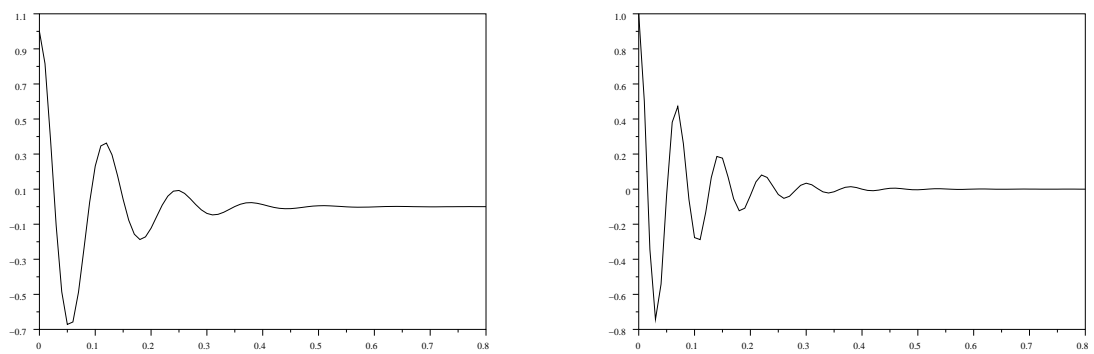

Figure 3: $u^{\epsilon}\left(x_{1}, 1\right) /\left|u^{\epsilon}(0,1)\right|$ for $\epsilon=10^{-3}$ and $10^{-5}$

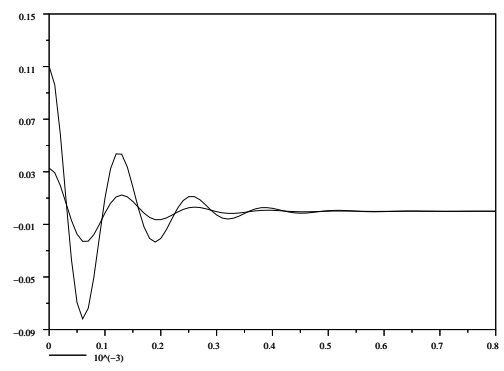

Figure 4: $u^{\epsilon}\left(x_{1}, 0.95\right)$ and $u^{\epsilon}\left(x_{1}, 0.75\right)$ for $\epsilon=10^{-3}$ 
In Figure 4 we displayed $u^{\epsilon}\left(x_{1}, 0.75\right)$ and $u^{\epsilon}\left(x_{1}, 0.95\right)$ for $\epsilon=10^{-3}$ and the loading $f\left(x_{1}, x_{2}\right)=\delta(x 1) \delta\left(x_{2}-0.75\right)$ the abscisse-axis is $\xi \in[0,8]$. The maximum of $\left|u^{\varepsilon}\left(x_{1}, 0.75\right)\right|$ and $\left|u^{\varepsilon}\left(x_{1}, 0.95\right)\right|$ are around 0.04 and 0.11 respectively. It is then apparent that the solution is much more singular in the vicinity of the boundary $\Gamma_{1}$ than on $x_{1}=0.75$ where the loading is applied. In fact, in the present situation, the singular behavior is somewhat "non local" as it is mainly localized in the vicinity of the boundary bearing the pathological boundary conditions rather than on the support of the loading.

Remark 17. It should be noted that these results are close to those of [18], [20]. It then appears that the singular behavior is not very dependent on the perturbation terms.

\section{An elliptic problem with "adapted" boundary condi- tions}

In this second part of the paper, we consider elliptic problems with "adapted" boundary conditions and we compare the obtained results with what was found in the first part. We will consider two kinds of loads, belonging and not belonging to the dual of the energy space of the limit problem.

Let $a_{1}$ be the bilinear form given by:

$$
a_{1}(u, v)=\int_{\Omega} \partial_{\alpha \beta} u \partial_{\alpha \beta} v \mathrm{~d} x .
$$

We shall study the variational problem

$$
\left\{\begin{array}{l}
\text { Find } u_{1} \in V_{a_{1}} \text { such that, } \forall v \in V_{a_{1}} \\
a_{1}\left(u_{1}, v\right)=\langle f, v\rangle
\end{array}\right.
$$

where the space $V_{a_{1}}$ is again the "energy space" with the essential boundary conditions

$$
v\left(x_{1}, 0\right)=\partial_{2} v\left(x_{1}, 0\right)=0,
$$

which is the completion with the norm $\|v\|_{a_{1}}=a_{1}(v, v)^{1 / 2}$ of the set of $H^{2}(\Omega)$ functions satisfying (5.3). The fact that $\|v\|_{a_{1}}$ is a norm follows easily from the relations $\left\|\partial_{\alpha \beta} v\right\|_{L^{2}}=0$ together with the boundary conditions (5.3). It is easily seen that this space is merely the subspace of $H^{2}(\Omega)$ satisfying (5.3).

When the load $f$ is in the dual space $V_{a_{1}}^{\prime}$, since $a_{1}$ is a symmetric form, it follows that problem (5.2) is equivalent to the minimization of the functional:

$$
\int_{\Omega} \sum_{\alpha, \beta}\left|\partial_{\alpha \beta} v\right|^{2} \mathrm{~d} x-2 \int_{\Omega} f v \mathrm{~d} x,
$$


over $V_{a_{1}}$. Furthermore, after a formal integration by parts, the classical formulation of problem (5.2) is:

$$
\left\{\begin{array}{l}
\triangle^{2} u_{1}=f \text { on } \Omega \\
u_{1}\left(x_{1}, 0\right)=\partial_{2} u_{1}\left(x_{1}, 0\right)=0, \forall x_{1} \in \mathbb{R} \\
\partial_{2}^{2} u_{1}\left(x_{1}, 1\right)=\partial_{2}\left(\triangle+\partial_{1}^{2}\right) u_{1}\left(x_{1}, 1\right)=0, \forall x_{1} \in \mathbb{R}
\end{array}\right.
$$

Contrary to the boundary value problem $(2.5)$, we observe that the boundary value problem (5.5) satisfies the Shapiro - Lopatinskii condition. This is obvious for the conditions on $x_{2}=0$ which are the Dirichlet conditions for $\Delta^{2}$. As for the conditions on $x_{2}=1$, we have: Considering the upper half plane $\left(x_{1}, x_{2}\right)$, $u_{1} \in V_{a_{1}}$ and the equation

$$
\triangle^{2} u_{1}\left(x_{1}, x_{2}\right)=\left(\partial_{1}^{4}+2 \partial_{1}^{2} \partial_{2}^{2}+\partial_{2}^{4}\right) u_{1}\left(x_{1}, x_{2}\right)=0,
$$

with the boundary conditions

$$
\partial_{2}^{2} u_{1}\left(x_{1}, 1\right)=\partial_{2}\left(\triangle+\partial_{1}^{2}\right) u_{1}\left(x_{1}, 1\right)=0,
$$

and taking the formal Fourier transform in the $x_{1}$ direction, this gives:

$$
\left(\xi^{4}-2 \xi^{2} \partial_{2}^{2}+\partial_{2}^{4}\right) \hat{u}_{1}\left(\xi, x_{2}\right)=0,
$$

with the boundary conditions

$$
\partial_{2}^{2} \hat{u}_{1}(\xi, 1)=\partial_{2}\left(2 \xi^{2}-\partial_{2}^{2}\right) \hat{u}_{1}(\xi, 1)=0 .
$$

The solutions of the equation which decay towards the domain are $\hat{u}_{1}\left(\xi, x_{2}\right)=$ $\left(A+B x_{2}\right) e^{-|\xi| x_{2}}$. Then, imposing the boundary conditions we see that $A=$ $B=0$. So, the Shapiro - Lopatinskii condition is satisfied and we have a classical elliptic problem.

We shall mainly consider the two loadings

$$
f=\delta\left(x_{1}\right) \delta\left(x_{2}-c\right)
$$

and

$$
f=\delta^{(4)}\left(x_{1}\right) \delta\left(x_{2}-c\right),
$$

where $\delta^{(4)}(x)$ is the fourth derivative of $\delta(x)$, as well as sometimes

$$
f=\delta\left(x_{1}\right) F\left(x_{2}\right)
$$

and

$$
f=\delta^{(4)}\left(x_{1}\right) F\left(x_{2}\right)
$$

with $F \in L^{2}(0,1)$. Obviously, (5.6) and (5.8) are in $V_{a_{1}}^{\prime}$, whereas (5.7) and (5.9) are not. In the first cas, existence and uniqueness of the solution follows from the Lax - Milgram theorem; nevertheless, in order to make comparisons with the previous sections, we shall give a (more or less) explicit construction of the solutions 
via Fourier transform, which reduces the problem to ordinary differential equations in $x_{2}$ depending on the parameter $\xi$. Obviously, the Fourier transforms of (5.6) and (5.7) are respectively

$$
\hat{f}\left(\xi, x_{2}\right)=\delta\left(x_{2}-c\right)
$$

and

$$
\hat{f}\left(\xi, x_{2}\right)=\xi^{4} \delta\left(x_{2}-c\right),
$$

Let us now go further into details.

We first consider (5.6). The Fourier transform of (5.5) with respect to $x_{1}$, yields to the following boundary value problem which depends on the parameter $\xi \in \mathbb{R}$

$$
\left\{\begin{array}{l}
\left(\partial_{2}^{2}-\xi^{2}\right)\left(\partial_{2}^{2}-\xi^{2}\right) \hat{u}_{1}\left(\xi, x_{2}\right)=\delta\left(x_{2}-c\right), \forall x_{2} \in(0,1) \\
\hat{u}_{1}(\xi, 0)=\partial_{2} \hat{u}_{1}(\xi, 0)=0 \\
\partial_{2}^{2} \hat{u}_{1}(\xi, 1)=\partial_{2}\left(\partial_{2}^{2}-2 \xi^{2}\right) \hat{u}_{1}(\xi, 1)=0 .
\end{array}\right.
$$

Recalling first that solutions of

$$
\left(\partial_{2}^{2}-\xi^{2}\right)\left(\partial_{2}^{2}-\xi^{2}\right) \hat{u}\left(\xi, x_{2}\right)=0
$$

are linear combinations of

$$
e^{|\xi| x_{2}}, e^{-|\xi| x_{2}}, x_{2} e^{|\xi| x_{2}}, x_{2} e^{-|\xi| x_{2}}
$$

we deduce that solutions of (5.12) are functions $\hat{u}_{1}\left(\xi, x_{2}\right)$ of the form

$$
\hat{u}_{1}\left(\xi, x_{2}\right)=\left\{\begin{array}{lll}
\hat{u}_{1}^{-}\left(\xi, x_{2}\right) & \text { if } & \left.x_{2} \in\right] 0, c[, \\
\hat{u}_{1}^{+}\left(\xi, x_{2}\right) & \text { if } & \left.x_{2} \in\right] c, 1[,
\end{array}\right.
$$

where $\hat{u}_{1}^{-}(\xi,),. \hat{u}_{1}^{+}(\xi,$.$) will be considered as defined on \mathbb{R}$ (i. e., we shall also consider the analytic continuation of them out of their definition domain) such that

$$
\left\{\begin{array}{l}
\hat{u}_{1}^{-}\left(\xi, x_{2}\right)=\left(C_{1}^{-}+C_{3}^{-} x_{2}\right) e^{|\xi| x_{2}}+\left(C_{2}^{-}+C_{4}^{-} x_{2}\right) e^{-|\xi| x_{2}} \\
\hat{u}_{1}^{+}\left(\xi, x_{2}\right)=\left(C_{1}^{+}+C_{3}^{+} x_{2}\right) e^{|\xi| x_{2}}+\left(C_{2}^{+}+C_{4}^{+} x_{2}\right) e^{-|\xi| x_{2}}
\end{array}\right.
$$

where $C_{k}^{-}, C_{k}^{+} \in \mathbb{C}$, for $k \in\{1,2,3,4\}$. The distribution $\delta\left(x_{2}-c\right)$ at the right hand side of (5.12) may be described in terms of the jumps and we see that $\hat{u}$ is a solution of

$$
\left(\partial_{2}^{2}-\xi^{2}\right)\left(\partial_{2}^{2}-\xi^{2}\right) \hat{u}\left(\xi, x_{2}\right)=\delta\left(x_{2}-c\right)
$$

when $\hat{u}^{-}$and $\hat{u}^{+}$satisfy

$$
\left\{\begin{aligned}
\hat{u}^{-}(\xi, c) & =\hat{u}^{+}(\xi, c) \\
\left(\hat{u}^{-}\right)^{\prime}(\xi, c) & =\left(\hat{u}^{+}\right)^{\prime}(\xi, c) \\
\left(\hat{u}^{-}\right)^{\prime \prime}(\xi, c) & =\left(\hat{u}^{+}\right)^{\prime \prime}(\xi, c) \\
\left(\hat{u}^{-}\right)^{\prime \prime \prime}(\xi, c)+1 & =\left(\hat{u}^{+}\right)^{\prime \prime \prime}(\xi, c)
\end{aligned}\right.
$$


A direct computation on the derivatives of $\hat{u}^{-}$and $\hat{u}^{+}$shows that

$$
\left\{\begin{array}{l}
C_{4}^{+}=C_{4}^{-}+\frac{e^{|\xi| c}}{4 \xi^{2}} \\
C_{3}^{+}=C_{3}^{-}+\frac{e^{-|\xi| c}}{4 \xi^{2}} \\
C_{2}^{+}=C_{2}^{-}+\frac{(1-|\xi| c) e^{|\xi| c}}{4|\xi|^{3}} \\
C_{1}^{+}=C_{1}^{-}-\frac{\left(1+|\xi| c e^{-|\xi| c}\right.}{4|\xi|^{3}}
\end{array}\right.
$$

Referring next to the boundary conditions of (5.12), it yields

$$
\left\{\begin{aligned}
C_{4}^{-}= & \frac{1}{3 e^{-|\xi|}+e^{|\xi|}}\left(2|\xi| C_{3}^{-}-\frac{3}{4 \xi^{2}} e^{|\xi|(c-1)}+\frac{-1+2|\xi|(1-c)}{4|\xi|^{2}} e^{|\xi|(1-c)}\right) \\
C_{3}^{-}= & \frac{1}{4 \xi^{2} e^{|\xi|}+\left(3+e^{2|\xi|}\right)\left(e^{-|\xi|}+3 e^{|\xi|}\right)}\left(\frac{1-2|\xi|(1-c)}{2|\xi|} e^{|\xi|(1-c)}-\frac{(1+2|\xi|(1-c))}{4 \xi^{2}} e^{|\xi|(c-1)}\right. \\
& \left.-\frac{3}{4 \xi^{2}} e^{|\xi|(3-c)}\right) \\
C_{2}^{-}= & -C_{1}^{-}=\frac{1}{2|\xi|}\left(C_{3}^{-}+C_{4}^{-}\right) .
\end{aligned}\right.
$$

and

$$
\begin{aligned}
\hat{u}^{+}\left(\xi, x_{2}\right)= & \hat{u}^{-}\left(\xi, x_{2}\right)+\frac{\left(x_{2}-c\right)}{2 \xi^{2}} \cosh \left(|\xi|\left(x_{2}-c\right)\right) \\
& -\frac{1}{2|\xi|^{3}} \sinh \left(|\xi|\left(x_{2}-c\right)\right),
\end{aligned}
$$

which gives the complete description of the Fourier transform.

We are now interested in the behavior of this Fourier transform for large $|\xi|$.

From the previous expressions, it easily follows that

$$
\left\{\begin{array}{l}
C_{4}^{-} \approx-\frac{c}{2 \xi \xi} e^{-|\xi| c} \\
C_{3}^{-} \approx-\frac{1}{4 \xi^{2}} e^{-|\xi| c} \\
C_{2}^{-}=-C_{1}^{-} \approx-\frac{c}{4|\xi|^{2}} e^{-|\xi| c}
\end{array}\right.
$$

Therefore, we obtain that for $0<x_{2}<c$ as $|\xi| \rightarrow \infty$,

$$
\hat{u}_{1}\left(\xi, x_{2}\right)=\hat{u}_{1}^{-}\left(\xi, x_{2}\right) \approx \frac{c-x_{2}}{4 \xi^{2}} e^{|\xi|\left(x_{2}-c\right)} .
$$

Similarly, for $c<x_{2}<1$ as $|\xi| \rightarrow \infty$, we get

$$
\hat{u}_{1}\left(\xi, x_{2}\right)=\hat{u}_{1}^{+}\left(\xi, x_{2}\right) \approx \frac{c-x_{2}}{4 \xi^{2}} e^{|\xi|\left(c-x_{2}\right)} .
$$

and for $x_{2}=c$ as $|\xi| \rightarrow \infty$, a direct computation gives that

$$
\hat{u}_{1}(\xi, c)=\hat{u}_{1}^{-}(\xi, c)=\hat{u}_{1}^{+}(\xi, c) \approx \frac{3}{8|\xi|^{3}},
$$


At this point, it will prove useful to recall very classical properties (see for instance [4] sect. 2.3 if necessary) relating smoothness of the function with behavior at infinity of its Fourier transform. Let $\varphi(x)$ be a function which is smooth for $x \in \mathbb{R}$ unless at $x=0$, where its derivatives have jumps $\left[\varphi^{n}\right]_{0}$. Then the Fourier transform has the asymptotic expansion for $|\xi| \rightarrow \infty$ :

$$
\hat{\varphi}(\xi) \simeq \sum_{n=0}^{\infty}(-i / \xi)^{n+1}\left[\varphi^{n}\right]_{0},
$$

(obviously, the case when the discontinuities of the function are located at $x=$ $\alpha \neq 0$ is deduced from the previous one by translation, and the factor $e^{i \alpha \xi}$ sould be included in the right hand side). Moreover, when the Fourier transform decays exponentially at infinity, the function is analytic.

Remark 18. It follows from (5.22), (5.23) and (5.24) and from the above considerations that $u_{1}\left(x_{1}, x_{2}\right)$ is analytic of $x_{1}$ for $x_{2} \neq c$, whereas for $x_{2}=c$ it is not smooth at the origin. This obviously agrees with regularity theory for elliptic equations; in fact $u_{1}$ is analytic of $x_{1}$ and $x_{2}$ unless at the origin, where it behaves as the fundamental solution of $\triangle^{2}$. This results should be compared with those of section 2 , where the "solution" with fixed $x_{2}$ is very singular of $x_{1}$, and even not a distribution.

Remark 19. We proceed similarly when the load is of the form: $f=\delta\left(x_{1}\right) F\left(x_{2}\right)$, with $F \in L^{2}(0,1)$. Indeed, in problem (5.12) the right hand side becomes $F\left(x_{2}\right)$. After an integration by parts, we obtain the variational formulation in $H_{\Gamma_{0}}^{2}(0,1)$ :

$$
\left\langle\partial_{2}^{2} \hat{u}_{1}, \partial_{2}^{2} v\right\rangle+2 \xi^{2}\left\langle\partial_{2} \hat{u}_{1}, \partial_{2} v\right\rangle+\xi^{4}\left\langle\hat{u}_{1}, v\right\rangle=\langle F, v\rangle,
$$

where $v$ is the test function. Then, defining the new unknown $\hat{w}_{1}=\xi^{4} \hat{u}_{1}$, the problem becomes

$$
\frac{1}{\xi^{4}}\left\langle\partial_{2}^{2} \hat{w}_{1}, \partial_{2}^{2} v\right\rangle+\frac{2}{\xi^{2}}\left\langle\partial_{2} \hat{w}_{1}, \partial_{2} v\right\rangle+\left\langle\hat{w}_{1}, v\right\rangle=\langle F, v\rangle,
$$

with the parameter $\xi$. Under the form (5.27), and considering $|\xi| \rightarrow \infty$, the coefficients $\xi^{-4}$ and $\xi^{-2}$ tend to 0 , so that the problem appears as a singular perturbation. The limit problem is merely $\left\langle\hat{w}_{1}, v\right\rangle=\langle F, v\rangle$, with variational formulation in $L^{2}(0,1)_{x_{2}}$. It then follows (the proof is standard, as in Lemma 3.3, for instance) that

$$
\hat{w}_{1}(\xi) \rightarrow F \quad \text { strongly in } L^{2}(0,1)_{x_{2}}
$$

when $|\xi|$ tends to $\infty$.

This means that $w_{1}\left(x_{1}\right)$ has a $\delta\left(x_{1}\right)$ singularity at the origin. This is in agreement with classical regularity theory for elliptic equations, since $\triangle^{2} w_{1}\left(x_{1}, x_{2}\right)=$ $\delta^{4}\left(x_{1}\right) F\left(x_{2}\right)$. Coming back to $u_{1}$, this gives that at the origin, $\partial_{1}^{4} u_{1}\left(x_{1}, x_{2}\right)$ has a $\delta\left(x_{1}\right)$ singularity at the origin. 
Let us now consider the case of a the load $f=\delta^{4}\left(x_{1}\right) \delta\left(x_{2}-c\right)$. Such a load does not belong to the dual space $V_{a_{1}}^{\prime}$, so that we can not start from the variational theory. In fact, as before, we explicitly construct the solution $\hat{u}_{1}$ using the Fourier transform and we obtain the same boundary value problem (5.12), except that the right hand-side term is multiplied by $\xi^{4}$. Clearly, the solution is merely $\xi^{4}$ times the previous one. It follows that for constant $x_{2}$ different from $c$ the solution decays exponentially as $|\xi| \rightarrow \infty$, whereas for $x_{2}=c$ :

$$
\hat{u}_{1}(\xi, c)=\hat{u}_{1}^{-}(\xi, c)=\hat{u}_{1}^{+}(\xi, c) \approx \frac{3|\xi|}{8},
$$

which tends to infinity with $|\xi|$. The solution $u_{1}\left(x_{1}, x_{2}\right)$ is obviously the fourth order derivative with respect to $x_{1}$ of the previous one.

Finally, the case $f=\delta^{(4)}\left(x_{1}\right) F\left(x_{2}\right)$ is done similarly to that of $f=\delta\left(x_{1}\right) F\left(x_{2}\right)$. We find that $\hat{u}_{1}(\xi) \rightarrow F$ strongly in $L^{2}(0,1)_{x_{2}}$ as $|\xi|$ tends to infinity, so that $u_{1}$ has a $\delta\left(x_{1}\right)$ singularity of intensity $F\left(x_{2}\right)$ at $x_{1}=0$.

\section{Singular perturbation of the elliptic problem with "adapted" boundary conditions}

We now consider the perturbated problem

$$
\left\{\begin{array}{l}
\text { Find } u_{1}^{\epsilon} \in V \text { such that, } \forall v \in V \\
a_{1}\left(u_{1}^{\epsilon}, v\right)+\epsilon^{2} b_{1}\left(u_{1}^{\epsilon}, v\right)=\langle f, v\rangle
\end{array}\right.
$$

where $a_{1}$ is given by (5.1) and

$$
b_{1}(u, v)=\int_{\Omega}\left(\partial_{1}^{3} u \partial_{1}^{3} v+\partial_{2}^{3} u \partial_{2}^{3} v\right) \mathrm{d} x .
$$

As in section 3 , for $\varepsilon>0$, the energy space $V$ is

$$
V=L^{2}\left(\mathbb{R}_{x_{1}} ; H_{\Gamma_{0}}^{3}(0,1)_{x_{2}}\right) \cap H^{3}\left(\mathbb{R}_{x_{1}} ; L^{2}(0,1)_{x_{2}}\right) .
$$

By a standard integration by parts, we obtain the classical formulation of the variational problem (6.1):

$$
\triangle^{2} u_{1}^{\epsilon}-\epsilon^{2}\left(\partial_{1}^{6}+\partial_{2}^{6}\right) u_{1}^{\epsilon}=f \text { on } \Omega,
$$

with the (essential) boundary conditions on $x_{2}=0$ :

$$
u_{1}^{\epsilon}\left(x_{1}, 0\right)=\partial_{2} u_{1}^{\epsilon}\left(x_{1}, 0\right)=\partial_{2}^{2} u_{1}^{\epsilon}\left(x_{1}, 0\right)=0, \forall x_{1} \in \mathbb{R},
$$

and the (natural) boundary conditions on $x_{2}=1$ :

$\epsilon^{2} \partial_{2}^{3} u_{1}^{\epsilon}\left(x_{1}, 1\right)=-\left(\partial_{2}^{2}+\epsilon^{2} \partial_{2}^{4}\right) u_{1}^{\epsilon}\left(x_{1}, 1\right)=\left(\partial_{2}\left(\triangle+\partial_{1}^{2}\right)+\epsilon^{2} \partial_{2}^{5}\right) u_{1}^{\epsilon}\left(x_{1}, 1\right)=0, \forall x_{1} \in \mathbb{R}$. 
Moreover since loads (5.6) and (5.7) belong to the space $V^{\prime}$, there exits a unique solution of the variational problem (6.1) for each load (5.8) and (5.9). The treatment of both loads is the same, and for simplicity we begin with $f=$ $\delta^{(4)}\left(x_{1}\right) F\left(x_{2}\right)$ with $F \in L^{2}(0,1)$. Taking the Fourier transform we obtain the equation with parameter $\xi$ :

$$
\left(\left(\partial_{2}^{2}-\xi^{2}\right)\left(\partial_{2}^{2}-\xi^{2}\right)-\epsilon^{2}\left(-\xi^{6}+\partial_{2}^{6}\right)\right) \hat{u}_{1}^{\epsilon}\left(\xi, x_{2}\right)=\xi^{4} F\left(x_{2}\right), \forall x_{2} \in(0,1)
$$

with the boundary conditions

$$
\begin{array}{r}
\hat{u}_{1}^{\epsilon}(\xi, 0)=\partial_{2} \hat{u}_{1}^{\epsilon}(\xi, 0)=\partial_{2}^{2} \hat{u}_{1}^{\epsilon}(\xi, 0)=0,(6.7) \\
\epsilon^{2} \partial_{2}^{3} \hat{u}_{1}^{\epsilon}(\xi, 1)=-\left(\partial_{2}^{2}+\epsilon^{2} \partial_{2}^{4}\right) \hat{u}_{1}^{\epsilon}(\xi, 1)=\left(\partial_{2}\left(\partial_{2}^{2}-2 \xi^{2}\right)+\epsilon^{2} \partial_{2}^{5}\right) \hat{u}_{1}^{\epsilon}(\xi, 1)=0 .(6.8)
\end{array}
$$

Let us divide (6.6) by $\xi^{4}$ and after an integration by parts, we get its variational form in $H_{\Gamma_{0}}^{3}(0,1)$ :

$$
\left(1+\epsilon^{2} \xi^{2}\right)\left\langle\hat{u}_{1}^{\epsilon}(\xi), v\right\rangle+\frac{2}{\xi^{2}}\left\langle\partial_{2} \hat{u}_{1}^{\epsilon}(\xi), \partial_{2} v\right\rangle+\frac{1}{\xi^{4}}\left\langle\partial_{2}^{2} \hat{u}_{1}^{\epsilon}(\xi), \partial_{2}^{2} v\right\rangle+\frac{\epsilon^{2}}{\xi^{4}}\left\langle\partial_{2}^{3} \hat{u}_{1}^{\epsilon}(\xi), \partial_{2}^{3} v\right\rangle=\langle F, v\rangle .
$$

We are now interested in properties of the solutions for small $\varepsilon$ and large $|\xi|$, to throw regularity properties of the inverse Fourier transform. In this context, we can do different things in (6.9). First, if we fix the value of $\epsilon$ and let $|\xi|$ go to infinity, we then easily deduce (as in the proof of (5.28)) that

$$
\hat{u}_{1}^{\epsilon}(\xi) \approx \frac{1}{\xi^{2}} \frac{1}{\epsilon^{2}} F
$$

or in other words that $\xi^{2} \hat{u}_{1}^{\epsilon}(\xi) \rightarrow \frac{F}{\epsilon^{2}}$ and this means that the second derivative $\partial_{1}^{2} u_{1}$ has a jump of value $\frac{F}{\epsilon^{2}}$ in 0 . We may also fix $\xi$ and let $\varepsilon \rightarrow 0$. Using the same techniques as in sect. 3 , we easily prove the convergence in $D^{\prime}\left(\mathbb{R}_{\xi} ; H_{\Gamma_{0}}^{2}(0,1)\right)$ towards the corresponding solution of the problem of section 5. All these properties are not very surprising nor very interesting.

Let us now consider another type of convergence. We consider again (6.9) as a variational problem depending on the parameters $\varepsilon$ and $\xi$. Let us then take $\lambda=\epsilon \xi$ as fixed and let $|\xi|$ go to infinity. In other words, we take $\varepsilon \rightarrow 0$ and $\xi=\lambda / \varepsilon$, where $\lambda$ is a fixed number. Then, using again the same method as in the prof of (5.28), we have:

Theorem 6.1. Let $\hat{u}_{1}^{\epsilon}(\xi)$ be the solution of (6.9). Taking $\varepsilon \rightarrow 0$ and $\xi=\lambda / \varepsilon$, where $\lambda$ is a fixed number, the following strong convergence holds:

$\hat{u}_{1}^{\epsilon}(\xi) \rightarrow \frac{F\left(x_{2}\right)}{\left(1+\lambda^{2}\right)}$, strongly in $L^{2}(0,1)_{x_{2}}$, as $|\xi| \rightarrow \infty$, with fixed values of $\lambda=\epsilon \xi$ 
Let us explain a little the meaning of this mathematical result. Passing to the limit in (6.10) amounts to neglecting in (6.9) the terms with factors $2 / \xi^{2}, 1 / \xi^{4}$ and $\varepsilon^{2} / \xi^{4}$. Under the hypothesis $\varepsilon=\lambda / \xi$ with fixed $\xi$, this amounts to neglecting $1 /|\xi|$ when it is smaller than $\varepsilon / \lambda$ with fixed $\lambda$, i. e. $1 /|\xi|$ smaller than $O(\varepsilon)$. In other words, taking the limit in (6.10) instead of $\hat{u}_{1}^{\epsilon}(\xi)$ amounts to neglecting the frequencies $|\xi|$ of order larger than $O\left(\varepsilon^{-1}\right)$ or, equivalently, the oscillations with wave length smaller than $O(\varepsilon)$. Within this approximation, we obtain

$$
\hat{u}_{1}^{\epsilon}(\xi) \approx \frac{F}{\left(1+\lambda^{2}\right)}=\frac{F}{\left(1+\epsilon^{2} \xi^{2}\right)} .
$$

Considering now the inverse Fourier transform, $\xi \rightarrow x_{1}$ and $\epsilon$ as a parameter, we see that $u_{1}^{\epsilon}\left(x_{1}\right) \approx \frac{1}{\epsilon} \varphi\left(\frac{x}{\epsilon}\right)$, where $\varphi(y)$ is the inverse Fourier transform of $\frac{F}{\left(1+z^{2}\right)}=$ $\varphi(z)$. A direct computation gives that $\varphi(y)=\frac{F}{2} e^{-|y|}$. Consequently, neglecting the oscillations of $\frac{1}{|\xi|}$ with respect with $\mathcal{O}(\epsilon)$, we obtain that

$$
u_{1 \text { approx }}^{\epsilon}\left(x_{1}, x_{2}\right) \approx \frac{1}{\epsilon} \frac{F\left(x_{2}\right)}{2} e^{-\left|\frac{x_{1}}{\epsilon}\right|} .
$$

The previous approximation (6.12) converges to the limit $\delta\left(x_{1}\right) F\left(x_{2}\right)$. This is a "boundary layer" near $x_{1}=0$, which coincides with the classical results of this kind of problem with a load $f$ that does not belong to $V_{a_{1}}^{\prime}$, see [17].

Obviously, the case of the applied load $f=\delta\left(x_{1}\right) F\left(x_{2}\right)$ is done similarly setting $\hat{w}_{1}^{\epsilon}\left(\xi, x_{2}\right)=\xi^{4} \hat{u}_{1}^{\epsilon}\left(\xi, x_{2}\right)$.

\section{References}

[1] S. Agmon, A. Douglis and L. NirenberG, Estimates near the boundary for solutions of elliptic partial differential equations satisfying general boundary conditiions, Communic. Pure. Applied Math., vol. 12, p. 623-727, 1959.

[2] R. Courant and D. Hilbert, Methods of mathematical physics, volume II, Interscience, New York, 1962.

[3] Y. V. EgOROV and B. W. SCHulze, Pseudodifferential operators, singularities and applications, Birkhauser, Berlin 1997.

[4] A. ERdelyi, Asymptotic expansions, Dover, New York, 1956.

[5] P. GÉrard and E. Sanchez Palencia, Sensitivity phenomena for certain thin elastic shells with edges. Math. Meth. Appl. Sci. 23 (2000), 379-399.

[6] S. G. Gindikin and L. R. Volevich, The Cauchy Problem, in Encyclopaedia of Mathematical Sciences, vol 32 (= Partial Differential Equations III), Springer, p. 1-86, 1991.

[7] I. M. Guelfand and G. E. ChIlov, Les distributions, Dunod, Paris, 1962. 
[8] D. HueT, Phénomènes de perturbation singulière dans les problèmes aux limites, Ann. Inst. Fourier, vol. 10, p. 61-150, 1960.

[9] T. KATO, Perturbation Theory for linear operators, Springer, Berlin, 1966.

[10] A. I. KомесH, Linear partial differential equations with constant coefficients, in Encyclopaedia of Mathematical Sciences, vol 31 (= Partial Differential Equations II), Springer, p. 121-255, 1994.

[11] J. L. LiOns, Perturbations singulières dans les problèmes aux limites et en contrôle optimal, Springer-Verlag, Berlin, (1973).

[12] J.-L. LiOnS and E. SANChEZ-PALEnCia, Problèmes sensitifs et coques élastiques minces, in Partial Differential Equations and Functional Analysis in memory of P. Grisvard, (ed. J. Cea, D. Chenais, G. Geymonat and J.-L. Lions), Birkhauser, p. 207-220, 1996.

[13] J. W. de RoEVER, Analytic representations and Fourier transforms of analytic functionals in $\mathcal{Z}^{\prime}$ carried by the real space, SIAM J. Math. Anal., vol. 9, p. 9961019, 1978.

[14] J. Sanchez Hubert and E. Sanchez Palencia, Vibration and coupling of continuous systems. Asymptotic methods, Springer, Berlin 1989.

[15] J. SAnchez Hubert and E. SAnchez Palencia, Coques élastiques minces. Propriétés asymptotiques, Masson, Paris 1997..

[16] E. Sanchez Palencia, Asymptotic and spectral properties of a class of singular - stiff problems, J. Math. Pures Appl. 71 (1992), 379-406.

[17] E. Sanchez Palencia, On the structure of layers for singularly perturbed equations in the case of unbounded energy, Control Optim. Calc. Var. 8 (2002), 941-963.

[18] E. Sanchez Palencia and C. A. de Souza, Complexification phenomenon in an example of sensitive singular perturbation, Comptes Rendus Mécanique, v. 332, p. 605-612, 2003.

[19] E. Sanchez Palencia and C. A. de Souza, Complexification phenomena in certain singular perturbations, in Fluid Mechanics: A volume in honor of Amable Liñan, F. J. HIguera, A. Jiménez and J. M. Vega (Eds.) CIMNE, Barcelona, 2004, p. 363 - 379.

[20] E. Sanchez Palencia and C. A. de Souza, Complexification in singular perturbations and their approximation, Int. J. Multiscale Comput. Engin., to appear, 2005 - 6.

[21] L. Schwartz, Les Distributions, Hermann, Paris, 1961. 